\title{
FATOR DE CORREÇÃO PARA A DISTRIBUIÇÃO DA DEVIANCE PARA DADOS DE PROPORÇÕES
}

\author{
ANA PAULA GOMES DA SILVA GIMENES \\ Engenheira Agrônoma
}

Orientador: Prof. Dr. JOSÉ EDUARDO CORRENTE

Dissertação apresentada à Escola Superior de Agricultura "Luiz de Queiroz", Universidade de São Paulo, para obtenção do título de Mestre em Agronomia, Área de Concentração:

Estatística e Experimentação Agronômica.

P I R A C I C A B A

Estado de São Paulo - Brasil

Agosto - 2000 


\section{ERRATA}

\begin{tabular}{|c|c|c|c|}
\hline Página & Linha & Onde se lê & Leia \\
\hline iv e $\mathrm{x}$ & & Deviance & Deviance \\
\hline $\mathrm{x}$ & 8 & for small sample ize. & for small sample size. \\
\hline 3 & 19 & Considerem-se & Considere-se \\
\hline 4 & 1 & onde cada $a_{i}$ é $A$ ou $\bar{A}$ & onde $a_{i} \in A$ ou $\bar{A}$ \\
\hline 6 & 11 & Taylor e Siqueira (1996) & Taylor et al. (1996) \\
\hline 8 & 5 & Família Binomial & família binomial \\
\hline 9 & 7 & Família Normal & família normal \\
\hline 10 & 10 & $h\left(x_{1}\right), \ldots, h\left(x_{n}\right)$ & $\left.h\left(x_{1}\right) \ldots h() x_{n}\right)$ \\
\hline 15 & 17,18 e 19 & $\mu$ e $\eta$ & $\boldsymbol{\mu}$ e $\boldsymbol{\eta}$ \\
\hline 16 & 4 & não existe a terceira afirmação. & \\
\hline 16 & 16 & para a função de ... & para o logaritmo da função de... \\
\hline 19 & 14 & ...modelos com $n$ parâmetros. & ...modelos com até $n$ parâmetros. \\
\hline 22 & 3 & de os cumulantes & dos cumulantes \\
\hline 24 & 10 & distribição & distribuição \\
\hline 24 & 22 & de se calcularem & que calculam \\
\hline 31 & 12 & Em estatística & Em Estatística \\
\hline 35 & 12 & $O\left(m^{3 / 2}\right)$ & $O\left(m^{-3 / 2}\right)$ \\
\hline 37 & 16 & $\cdots=2 \sum_{i=1}^{n} \cdots$ & $\ldots=2 \sum_{i=1}^{n} \sum_{y_{i}=1}^{m-1} \ldots$ \\
\hline 47 & 2 & $Y$ em $E(D(\mathbf{Y}, \hat{\mu}))=\ldots$ & $\boldsymbol{y}$ em $\boldsymbol{E}(D(\mathbf{Y}, \hat{\boldsymbol{\mu}}))=\ldots$ \\
\hline 49 & 5 & como mostram as Figuras 3,4 e 5 & como mostram as Figuras 3 e 4 \\
\hline 55 & 5 & $\ldots \operatorname{com} m=10$ e 20 e $p=0,1,0,2,0,3$ e 0,4 & $\begin{array}{l}m=10 \text { e } p=0,1,0,2,0,3 \text { e } 0,4 \\
m=20 \text { e } p=0,1 \text { e } 0,2, m=30,40 \\
\text { e } 50 \text { e } p=0,1\end{array}$ \\
\hline 60 & 10 & como mostram as Figuras 20 e 21 & como mostram as Figuras 20 a 22 \\
\hline
\end{tabular}


Dados Internacionais de Catalogação na Publicação (CIP)

DIVISÃO DE BIBLIOTECA E DOCUMENTAÇÃO - Campus "Luiz de QueirOz"/USP

Gimenes, Ana Paula Gomes da Silva

Fator de correção para a distribuição da deviance para dados de proporçōes / Ana

Paula Gomes da Silva Gimenes. - - Piracicaba, 2000.

$63 \mathrm{p}$.

Dissertação (mestrado) - - Escola Superior de Agricultura Luiz de Queiroz, 2000. Bibliografia.

1. Análise de variância 2. Distribuição binomial 3. Modelo matemático 4. Série de Taylor 5. Simulação I. Título

CDD 519.532

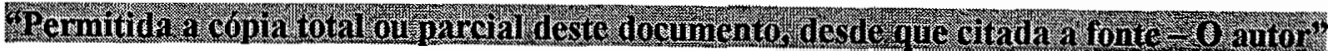




\section{Agradecimentos}

Ao meu pai e à minha mãe, pela confiança, apoio e colaboração.

Aos professores e funcionários do Departamento de Ciências Exatas (ex- Matemática e Estatística) da ESALQ/USP pela colaboração nos momentos importantes desta caminhada.

Ao Prof. Dr. José Eduardo Corrente, meu orientador, cuja paciência, cooperação e apoio foram fundamentais.

Ao meu filho Diogo, eu dedico.

À meu esposo, Milton, pela cooperação. 


\section{SUMÁRIO}

LISTA DE FIGURAS v v

RESUMO viii

SUMMARY $\mathbf{x}$

1 INTRODUÇÃO

2 REVISÃO DE LITERATURA 3

2.1 A Distribuição Binomial . . . . . . . . . . . . . . . . . . . 3

2.2 Famílias Exponenciais $\ldots \ldots \ldots \ldots 7$

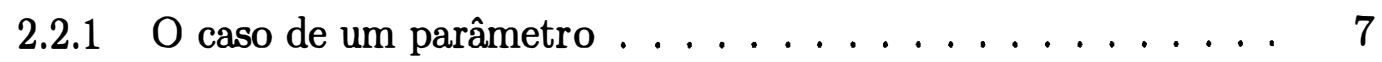

2.2 .2 O caso de $k$ parâmetros $\ldots \ldots \ldots \ldots$

2.3 Modelos Lineares Generalizados . . . . . . . . . . . . . . . . . . . . 11

2.3 .1 Seleção do Modelo . . . . . . . . . . . . . . . . . . . . 12

2.3 .2 Estimação . . . . . . . . . . . . . . . . . . . . . . 13

2.3 .3 Predição . . . . . . . . . . . . . . . . . . . . . . 13

2.3.4 Os componentes de um Modelo Linear Generalizado . . . . . . 14

2.4 Medindo a qualidade do ajuste . . . . . . . . . . . . . . . . . . . . 19

2.5 Correção de Bartlett . . . . . . . . . . . . . . . . . . . . . . 21

2.6 Propostas de Cordeiro (1986) $\ldots \ldots \ldots \ldots$

2.7 Proposta de Cox e Snell (1968) para Transformação da Variável Aleatória 24

2.8 Desenvolvimentos Assintóticos - Série de Taylor . . . . . . . . . . 26

2.8.1 Série de Taylor para funções de uma variável . . . . . . . . . 28

2.8.2 Série de Taylor para funções de Duas ou mais Variáveis . . . . 28

3 METODOLOGIA $\quad 34$

3.1 A deviance para a distribuição binomial . . . . . . . . . . . . . . . . 34 
3.2 Expansão da Deviance sem correção . . . . . . . . . . . . . . . . . 35

3.2 .1 Para uma variável . . . . . . . . . . . . . . . . . 35

$3.2 .2 \quad$ Para $n$ variáveis . . . . . . . . . . . . . . . . . 37

3.3 Expansão da Deviance considerando o Fator de Correção . . . . . . . 41

3.3.1 Para uma Variável . . . . . . . . . . . . . . . . . . . . 41

3.3.2 Para $n$ Variáveis . . . . . . . . . . . . . . . 43

4 RESULTADOS E DISCUSSÃO $\quad 47$

4.1 Estudo do Fator de Correção . . . . . . . . . . . . . . . . . . . . 60

5 CONCLUSÃo $\quad 61$

REFERÊNCIAS BIBLIOGRÁFICAS 


\section{LISTA DE FIGURAS}

1 Gráfico da deviance da distribuição binomial para o modelo de média

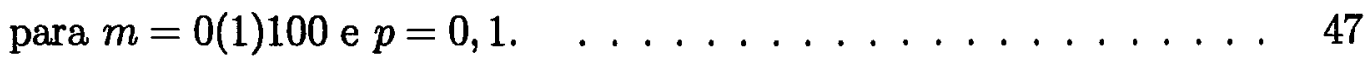

2 Gráfico da deviance da distribuição binomial para o modelo de média para $m=0(1) 100$ e $p=0,2 \ldots \ldots \ldots \ldots \ldots$

3 Gráfico da deviance da distribuição binomial para o modelo de média para $m=0(1) 50$ e $p=0,3 \ldots \ldots \ldots \ldots$

4 Gráfico da deviance da distribuição binomial para o modelo de média para $m=0(1) 50$ e $p=0,4 \ldots \ldots \ldots \ldots \ldots$

5 Gráfico da deviance da distribuição binomial para o modelo de média com e sem o fator de correção para $m=0(1) 100$ e $p=0,1$. ( $\ldots$ - . ... = deviance sem correção, - - - _ - - = deviance com correção)

6 Gráfico da deviance da distribuição binomial para o modelo de média com e sem o fator de correção para $m=0(1) 100$ e $p=0,2 .(-\ldots$ - - - = deviance sem correção, - - - - - = deviance com correção) .

$7 \quad$ Gráfico da deviance da distribuição binomial para o modelo de média com e sem o fator de correção para $m=0(1) 100$ e $p=0,3 .(-\cdots$ - - = deviance sem correção, - - - - - - = deviance com correção) .

8 Gráfico da deviance da distribuição binomial para o modelo de média com e sem o fator de correção para $m=0(1) 50$ e $p=0,3 .(-\ldots \ldots$ - = deviance sem correção, - - - - - - = deviance com correção) . .

9 Gráfico da deviance da distribuição binomial para o modelo de média com e sem o fator de correção para $m=0(1) 50$ e $p=0,4 .(\ldots \ldots$ - $\ldots$ - = deviance sem correção, $\ldots+\ldots$ 
10 Gráfico da deviance da distribuição binomial para o modelo de média com e sem o fator de correção $a=0,73$ para $m=0(1) 100$ e $p=0,1$. (- . . . . . . - deviance sem correção, $\ldots-\ldots-\ldots-$ = deviance com correção $\ldots \ldots \ldots \ldots \ldots \ldots \ldots \ldots \ldots \ldots \ldots$

11 Gráfico da deviance da distribuição binomial para o modelo de média com e sem o fator de correção $a=0,73$ para $m=0(1) 50$ e $p=0,2$. (- - . . . - - = deviance sem correção, $-\ldots-\cdots=$ deviance com correção $\ldots \ldots \ldots \ldots \ldots \ldots \ldots \ldots \ldots \ldots \ldots$

12 Gráfico da deviance da distribuição binomial para o modelo de média com e sem o fator de correção $a=0,73$ para $m=0(1) 30$ e $p=0,3$. (- - . - - - - = deviance sem correção, - - - - - = deviance com correção $\ldots \ldots \ldots \ldots \ldots \ldots \ldots \ldots \ldots \ldots \ldots$

13 Gráfico da deviance da distribuição binomial para o modelo de média com e sem o fator de correção $a=0,73$ para $m=0(1) 20$ e $p=0,4$. (- - - . - - = deviance sem correção, - - - - - = deviance com correção) . . . . . . . . . . . . . . . . . . . . . . . . .

14 QQ-plots dos valores da deviance da distribuição binomial contra os valores da qui-quadrado com 1 g.l. para o modelo de média sem e com o fator de correção para $m=10$ e $p=0,1 \ldots \ldots \ldots \ldots$. . . .

15 QQ-plots dos valores da deviance da distribuição binomial contra os valores da qui-quadrado com 1 g.l. para o modelo de média sem e com o fator de correção para $m=10$ e $p=0,2 \ldots \ldots \ldots \ldots$

16 QQ-plots dos valores da deviance da distribuição binomial contra os valores da qui-quadrado com 1 g.l. para o modelo de média sem e com o fator de correção para $m=10$ e $p=0,3 \ldots \ldots \ldots 6$

17 QQ-plots dos valores da deviance da distribuição binomial contra os valores da qui-quadrado com 1 g.l. para o modelo de média sem e com o fator de correção para $m=10$ e $p=0,4 \ldots \ldots \ldots$. . . . . . 57 
18 QQ-plots dos valores da deviance da distribuição binomial contra os valores da qui-quadrado com 1 g.l. para o modelo de média sem e com o fator de correção para $m=20$ e $p=0,1 \ldots \ldots \ldots \ldots \ldots \ldots$

19 QQ-plots dos valores da deviance da distribuição binomial contra os valores da qui-quadrado com 1 g.l. para o modelo de média sem e com o fator de correção para $m=20$ e $p=0,2 \ldots \ldots \ldots \ldots$

20 QQ-plots dos valores da deviance da distribuição binomial contra os valores da qui-quadrado com 1 g.l. para o modelo de média sem e com o fator de correção para $m=30$ e $p=0,1 \ldots \ldots \ldots \ldots \ldots$

21 QQ-plots dos valores da deviance da distribuição binomial contra os valores da qui-quadrado com 1 g.l. para o modelo de média sem e com o fator de correção para $m=40$ e $p=0,1 \ldots \ldots \ldots \ldots$

22 QQ-plots dos valores da deviance da distribuição binomial contra os valores da qui-quadrado com 1 g.l. para o modelo de média sem e com o fator de correção para $m=50$ e $p=0,1 \ldots \ldots \ldots \ldots$ 


\title{
FATOR DE CORREÇÃO PARA A DISTRIBUIÇÃO DA DEVIANCE PARA DADOS DE PROPORÇÕES
}

\author{
Autor: ANA PAULA GOMES DA SILVA GIMENES \\ Orientador: Prof. Dr. JOSÉ EDUARDO CORRENTE
}

\section{RESUMO}

A análise de dados de proporções apresenta, em geral, certas dificuldades uma vez que a distribuição subjacente a tais dados pode ser considerada binomial, que não segue as pressuposições básicas para o ajuste de um modelo matemático. Algumas transformações são sugeridas, mas nem sempre bons resultados são obtidos.

No enfoque de modelos lineares generalizados, a estatística que mede a qualidade do ajuste do modelo para os dados é chamada deviance. Ocorre que a distribuição da deviance é desconhecida. No entanto, para dados com distribuição binomial, pode-se aproximar a distribuição da deviance por uma distribuição $\chi^{2}$, mas tal aproximação não é boa para tamanhos pequenos de amostra. Para melhorar essa aproximação, alguns fatores de correção para os dados são sugeridos, mas os resultados obtidos ainda não são bons para pequenas amostras. Assim, o objetivo deste trabalho é propor um novo fator de correção para os dados seguindo uma distribuição binomial, de modo a se obter uma melhora na distribuição da deviance para qualquer tamanho de amostra. Para isto, adiciona-se uma constante à variável resposta e, através do valor esperado da deviance, calcula-se tal constante de modo a reduzir o erro cometido na aproximação. 
Simulações da distribuição binomial e o cálculo da deviance são feitos e QQ-plots são utilizados para a comparação com a distribuição qui-quadrado. 


\title{
CORRECTION FACTOR FOR THE DISTRIBUTION OF THE DEVIANCE FOR PROPORTION DATA
}

\author{
Author: ANA PAULA GOMES DA SILVA GIMENES \\ Adviser: Prof. Dr. JOSÉ EDUARDO CORRENTE
}

\section{SUMMARY}

Analysis of proportion data presents, in general, some problems once the underlying distribution of these data can be considered as a binomial, which does not follow the basic assumptions to fit a model. Some tranformations can be suggested, but good results are not always obtained. In the approach of the Generalized Linear Models, the deviance is the statistics that measures the goodness of fit, but its distribution is unknown. Furthermore, considering binomial distribution data, it is possible to approximate the distribution of the deviance for a chi-square distribution, but such approximation is not good for small sample ize. In order of improve this approximation, corrections for the data are suggested, but the results are not good yet. Then, the aim of this work is to propose a new correction factor for data following a binomial distribution in order to obtain an improvement in the distribution of the deviance for any sample size. For this, just adding a constant at the response variable and, through the expected value of the deviance, such constant is obtained in order to reduce the error in the aproximation. Simulations of the binomial distribution are made to calculate the deviance with and without the correction and QQ-plots are used to compare with the chi-square distribution. 


\section{INTRODUÇÃO}

Dados de proporções, em geral, podem ser supostos como provenientes de uma distribuição binomial e nesses casos, é comum fazer uma transformação estabilizadora da variância a fim de validar as pressuposições básicas para o ajuste do modelo a ser analisado.

Quando dados de proporções são coletados seguindo algum tipo de delineamento experimental, pode-se utilizar o enfoque de Modelos Lineares Generalizados para se proceder à análise utilizando-se a deviance, que mede a qualidade do ajuste para o modelo considerado. A deviance, é proporcional à diferença do logaritmo da função de verossimilhança do modelo superparametrizado em relação ao logaritmo da função de verossimilhança do modelo sob pesquisa.

A distribuição da deviance é desconhecida, mas, para alguns casos particulares como no caso da distribuição binomial, pode-se mostrar que sua distribuição é aproximadamente $\chi^{2}$ com $n-p$ graus de liberdade, onde $n$ é o número de observações e $p$ é o número de parâmetros envolvidos no modelo sob pesquisa. Tal aproximação apresenta um resto da ordem de $O\left(m^{-1}\right)$, onde $m$ representa o número de realizações de um ensaio binomial. Para reduzir o erro na aproximação da distribuição da deviance, alguns fatores de correção para essa distribuição têm sido propostos por vários autores, sem muito sucesso. Esses fatores de correção surgem através de procedimentos de testes de hipóteses, ou por correções no valor esperado da deviance. Acontece que tais fatores apresentam problemas quando se trata de pequenas amostras e nesses casos, tais correções ainda não são eficazes para melhorar a aproximação da distribuição da deviance a uma distribuição de $\chi^{2}$.

Assim, o objetivo deste trabalho é apresentar um novo fator de correção 
de modo a melhorar a aproximação da distribuição da deviance a uma distribuição $\chi^{2}$. A obtenção de tal fator será feita adicionando uma constante à variável resposta que possui uma distribuição binomial e, através da expansão em série de Taylor do valor esperado da deviance, calcula-se esse valor de modo a zerar os termos de ordem $m^{-1}$. Através de dados simulados, os gráficos do valor esperado da deviance para diversos valores de $m$ serão mostrados e QQ-plots são apresentados para verificar a adequacidade da distribuição $\chi^{2}$ à distribuição da deviance calculada para as variáveis resposta corrigidas. 


\section{REVISÃO DE LITERATURA}

\subsection{A Distribuição Binomial}

Segundo Johnson e Kotz (1969), a distribuição binomial é obtida da seguinte maneira: considere $m$ realizações independentes de um experimento dicotômico $\mathcal{E}$. Suponha que os resultados possíveis de $\mathcal{E}$ sejam $A$ e $\bar{A}$ com probabilidades $p$ e $(1-p)$, respectivamente, e sua distribuição é dita ser binomial com parâmetros $m$ e $p$.

A distribuição binomial é definida em termos da expansão do binômio de Newton da forma $(q+p)^{m}$, onde $q+p=1, p>0, q>0$ e $m$ é um inteiro positivo. $\mathrm{O}(k+1)$-ésimo termo na expansão de $(q+p)^{m}$, para $k=0,1, \ldots, m$, é

$$
\left(\begin{array}{c}
m \\
k
\end{array}\right) p^{k} q^{m-k}=\frac{m !}{k !(m-k) !} p^{k} q^{m-k}
$$

Assim, pode-se expressar a distribuição binomial de uma variável aleatória $X$, denotada por $X \sim b(m, p)$, da seguinte forma:

$$
P[A \text { ocorrer } k \text { vezes }]=P[X=k]=p(k)=\left(\begin{array}{c}
m \\
k
\end{array}\right) p^{k} q^{m-k}
$$

para $k=0,1,2, \ldots, m$.

Meyer (1984) apresenta a seguinte definição para a distribuição binomial: considere um experimento $\mathcal{E}$ e seja $A$ algum evento associado a $\mathcal{E}$. Admite-se que $P(A)=p$ e, consequentemente, $P(\bar{A})=1-p$, onde $\bar{A}$ é o conjunto complementar de $A$. Considerem-se $m$ repetições de $\mathcal{E}$. Daí, o espaço amostral será formado 
por todas as sequências possíveis $\left\{a_{1}, a_{2}, \ldots, a_{m}\right\}$, onde cada $a_{i}$ é $A$ ou $\bar{A}$, dependendo do que tenha ocorrido, $A$ ou $\bar{A}$, na $i$-ésima repetição de $\mathcal{E}$. (Existem $2^{m}$ dessas sequências.) Além disso, suponha-se que $P(A)=p$ permaneça a mesma para todas as realizações de $\mathcal{E}$. A variável aleatória $X$ será assim definida: $X=$ número de vezes que o evento $A$ tenha ocorrido. Denomina-se $X$ de variável aleatória binomial, com parâmetros $m$ e $p$. Seus valores possíveis são, evidentemente, $0,1,2, \ldots, m$ e a probabilidade de ocorrência de $k$ eventos é dada como em (1).

Murteira (1990) relata que a distribuição binomial é um modelo probabilístico adequado para descrever os processos em que se realizam repetidas provas de Bernoulli, isto é, sucessões de experiências aleatórias independentes em cada uma das quais observa-se a realização, ou não, de um determinado evento $A$, com probabilidade $P(A)=p$, constante de experiência para experiência. A ocorrência de $A$ será chamada de "sucesso"; a ocorrência do contrário, $\bar{A}$, que tem probabilidade $P(\bar{A})=1-p=q$, diz-se um "insucesso" ou "fracasso".

Os diferentes valores de $m$ (inteiro positivo) e de $p(0<p<1)$ correspondem a diferentes formas da distribuição; assim, $m$ e $p$ são chamados parâmetros da distribuição binomial.

As probabilidades de observar $X$, respectivamente, igual a $0,1,2, \ldots, m$ são:

$$
(1-p)^{m}, \quad m p(1-p)^{m-1}, \quad\left(\begin{array}{c}
m \\
2
\end{array}\right) p^{2}(1-p)^{m-2}, \quad \cdots, \quad p^{m}
$$

i.e, os termos sucessivos do desenvolvimento do binômio,

$$
[(1-p)+p]^{m}=1
$$

de onde vem que

$$
\sum_{k=0}^{m} P(X=k)=\sum_{k=0}^{m} p(k)=\sum_{k=0}^{m}\left(\begin{array}{c}
m \\
k
\end{array}\right) p^{k}(1-p)^{m-k}=1
$$


definindo uma verdadeira distribuição de probabilidade.

A função de distribuição acumulada correspondente à função probabilidade dada em (1) é :

$$
F(x)=P(X \leq x)=\sum_{k: k \leq x}\left(\begin{array}{c}
m \\
k
\end{array}\right) p^{k}(1-p)^{m-k}
$$

As funções característica e geradora de momentos da distribuição binomial são definidas por

$$
\phi(t)=\left(q+p e^{i t}\right)^{m} \quad \text { e } \quad M_{X}(t)=\left(q+p e^{t}\right)^{m}
$$

Usando-se essas funções, pode-se mostrar que

$$
\begin{gathered}
E(X)=m p \\
E\left(X^{2}\right)=m p+m(m-1) p^{2} \\
E\left(X^{3}\right)=m p+3 m(m-1) p^{2}+m(m-1)(m-2) p^{3} \\
E\left(X^{4}\right)=m p+7 m(m-1) p^{2}+6 m(m-1)(m-2) p^{3}+m(m-1)(m-2)(m-3) p^{4}
\end{gathered}
$$

e assim por diante.

Além disso, pode-se calcular os momentos centrais da distribuição binomial, que são dados por

$$
\begin{gathered}
E(X-E(X))^{2}=\operatorname{var}(X)=m p q \\
E(X-E(X))^{3}=m p q(q-p) \\
E(X-E(X))^{4}=3(m p q)^{2}+m p q(1-6 p q)
\end{gathered}
$$


$\mathrm{Na}$ presença de algum delineamento experimental e as pressuposições básicas para uma análise de variância clássica não são satisfeitas, principalmente devido à heterogeneidade de variâncias.

Algumas vezes uma transformação nesses dados pode ser utilizada a fim de estabilizar a variância. Cox e Snell (1981) comentam sobre a existência de três métodos para se fazer transformações, como segue:

i) uma transformação não linear de uma variável resposta efetivamente contínua para uma nova forma;

ii) uma transformação não linear do parâmetro, que é usualmente um valor esperado, numa representação da variação sistemática;

iii) uma transformação não linear de uma variável explanatória. Taylor e Siqueira (1996) relatam sobre o aumento da variância, ao adicionar parâmetros utilizando-se um modelo não linear, citando também que essa transformação apresenta várias controvérsias.

Em (i), pode-se supor que algumas formas transformadas da variável resposta satisfazem um modelo linear teoricamente normal, mas na prática isso nem sempre é possível. Quando se determina uma transformação, deseja-se encontrar uma estrutura linear simples, variância constante e distribuições normais. No caso de ocorrer um conflito entre essas estruturas, deve-se concentrar atenção na análise dos dados não transformados.

Em (ii) a transformação não linear não é aplicada à variável resposta, mas em um parâmetro que normalmente é a média. Assim, analisando dados binários, a resposta esperada é a probabilidade de sucesso, e uma importante família de modelos é obtida supondo que

$$
\log \left\{\frac{\text { prob. sucesso }}{\text { prob. fracasso }}\right\}
$$


resulta num modelo linear.

A distinção entre (i) e (ii) é ilustrado no contexto da teoria normal supondo-se que:

a) alguma função $g\left(Y_{i}\right)$ da resposta $Y_{i}$, por exemplo, $g\left(Y_{i}\right)=\log Y_{i}$, é normalmente distribuída com variância constante, e com a média contendo uma estrutura simples em termos de variáveis explanatórias;

b) $Y_{i}$ é normalmente distribuída com variância constante e que alguma função $g\left\{E\left(Y_{i}\right)\right\}$ de sua média, por exemplo, $\log E\left(Y_{i}\right)$, tem estrutura simples em termos de variáveis explanatórias.

Nesse contexto particular, (a) é o procedimento mais usado sempre.

\subsection{Famílias Exponenciais}

\subsubsection{O caso de um parâmetro}

Seja $X$ uma variável aleatória com uma distribuição $P_{\theta}$, onde $\theta$ é um parâmetro unidimensional, pertencente ao espaço paramétrico $\Theta$. Segundo Bickel e Doksum (1977), a família de distribuições de um modelo $\left\{P_{\theta}: \theta \in \Theta\right\}$, é chamada familia exponencial a um parâmetro, se existem funções de valores reais $c(\theta), d(\theta)$ em $\Theta$, funções de valores reais $T$ e $S$ em $\mathcal{R}^{n}$, e um conjunto $A \subset \mathcal{R}^{n}$ tal que a função de densidade (ou freqüência) $p(x, \theta)$ de $P_{\theta}$ pode ser escrita por:

$$
p(x, \theta)=\{\exp [c(\theta) T(x)+d(\theta)+S(x)]\} I_{A}(x)
$$

em que $I_{A}$ é uma função indicadora dos valores possíveis da variável aleatória $X$ pertencentes a um conjunto $A$. Note que as funções $c, d, S$ e $T$ não são únicas e o conjunto $A$ não depende do parâmetro $\theta$. 
Numa família exponencial a um parâmetro, a estatística $T(X)$ é suficiente para $\theta$. Isto é claro desde que é necessário somente identificar $e^{c(\theta) T(x)+d(\theta)}$ com $g(T(x), \theta)$ e $[\exp S(x)] I_{A}(x)$ com $h(x)$ no Teorema da Fatorização de Fisher-Neyman. Neste caso, $T(X)$ é a estatística suficiente natural da familia.

A Família Binomial é um exemplo de família exponencial a um parâmetro. Suponha que $X$ tem uma distribuição $b(m, \theta), 0<\theta<1$. Então

$$
p(x, \theta)=\left(\begin{array}{c}
m \\
x
\end{array}\right) \theta^{x}(1-\theta)^{m-x} I_{A}(x)
$$

onde $A=\{0,1, \ldots, m\}$. Reescrevendo (3) obtém-se

$$
p(x, \theta)=\left\{\exp \left[x \log \left(\frac{\theta}{1-\theta}\right)+m \log (1-\theta)+\log \left(\begin{array}{c}
m \\
x
\end{array}\right)\right]\right\} I_{A}(x)
$$

Daí, a família de distribuições de $X$ é uma família exponencial com um parâmetro, sendo

$$
\begin{gathered}
c(\theta)=\log \left(\frac{\theta}{1-\theta}\right), \quad d(\theta)=m \log (1-\theta), \\
T(x)=x, \quad S(x)=\log \left(\begin{array}{c}
m \\
x
\end{array}\right) .
\end{gathered}
$$

Bickel e Doksum (1977) ainda mostram que existe uma extensão natural da família exponencial com um parâmetro para $n$ variáveis.

\subsubsection{O caso de $k$ parâmetros}

Novamente, para a variável aleatória $X$ com distribuição $P_{\boldsymbol{\theta}}, \boldsymbol{\theta}$ agora um parâmetro $k$-dimensional, pertencente a um espaço paramétrico $\Theta$, uma família de distribuições $\left\{P_{\boldsymbol{\theta}}: \boldsymbol{\theta} \in \Theta\right\}$ é chamada de familia exponencial com $k$ parâmetros, se existem funções reais $c_{1}, \ldots, c_{k}$, e $d$ de $\boldsymbol{\theta}$, funções de valores reais $T_{1}, \ldots, T_{k}, S$ em 
$\mathcal{R}^{n}$ e um conjunto $A \subset \mathcal{R}^{n}$ tal que as funções de densidade (ou freqüências) de $P(\theta)$ possam ser escritas como

$$
p(x, \boldsymbol{\theta})=\left\{\exp \left[\sum_{i=1}^{k} c_{i}(\boldsymbol{\theta}) T_{i}(x)+d(\boldsymbol{\theta})+S(x)\right]\right\} I_{A}(x) .
$$

$\mathrm{O}$ vetor $\mathbf{T}(X)=\left(T_{1}(X), \ldots, T_{k}(X)\right)$ é uma estatística conjuntamente suficiente para os $k$ parâmetros envolvidos na distribuição. Na verdade, $\mathbf{T}(X)$ é a estatística suficiente natural da família.

A Família Normal com Média e Variância desconhecidas é um exemplo de família exponencial a dois parâmetros. Suponha que $X$ tenha distribuição normal com parâmetros $\mu$ e $\sigma^{2}$. Neste caso, $\Theta=\left\{\left(\mu, \sigma^{2}\right):-\infty<\mu<\infty, \sigma^{2}>0\right\}$ com $\boldsymbol{\theta}=\left(\mu, \sigma^{2}\right)$. A função de densidade de $p(x, \boldsymbol{\theta})$ é escrita como

$$
\begin{aligned}
p(x, \boldsymbol{\theta}) & =\frac{1}{\sqrt{2 \pi \sigma^{2}}} \exp \left\{\frac{-1}{2 \sigma^{2}}(x-\mu)^{2}\right\} \\
& =\exp \left[\frac{\mu}{\sigma^{2}} x-\frac{x^{2}}{2 \sigma^{2}}-\frac{1}{2}\left(\frac{\mu^{2}}{\sigma^{2}}+\ln \left(2 \pi \sigma^{2}\right)\right)\right]
\end{aligned}
$$

que corresponde à família exponencial com dois parâmetros, sendo

$$
\begin{aligned}
& c_{1}(\boldsymbol{\theta})=\frac{\mu}{\sigma^{2}}, \quad T_{1}(x)=x, \quad c_{2}(\boldsymbol{\theta})=-\frac{1}{2 \sigma^{2}}, \quad T_{2}(x)=x^{2}, \\
& d(\boldsymbol{\theta})=-\frac{1}{2}\left(\frac{\mu^{2}}{\sigma^{2}}+\log \left(2 \pi \sigma^{2}\right)\right), \quad S(x)=0, \quad \text { e } \quad A=\mathcal{R} .
\end{aligned}
$$

No caso de se observar uma amostra $\mathbf{X}=\left(X_{1}, \ldots, X_{m}\right)$ de uma população $\mathcal{N}\left(\mu, \sigma^{2}\right)$, as estatísticas conjuntamente suficientes naturais serão $\left(\sum_{i=1}^{m} X_{i}\right.$, $\left.\sum_{i=1}^{m} X_{i}^{2}\right)$.

Roussas (1973) mostra a seguinte definição para o caso da família exponencial com um parâmetro: uma grande classe de funções de densidade de probabilidades (ou distribuição de freqüências) dependem de um parâmetro de valor real $\theta$ da seguinte forma: 


$$
f(x, \theta)=C(\theta) e^{Q(\theta) T(x)} h(x), \quad x \in \mathcal{R}, \quad \theta \in \Omega(\subseteq \mathcal{R})
$$

onde $C(\theta)>0, \theta \in \Omega$ e também $h(x)>0$ para $x \in S$, o conjunto de positividade de $f(x, \theta)$, que é independente de $\theta$. Segue que

$$
C^{-1}(\theta)=\sum_{x \in S} e^{Q(\theta) T(x)} h(x)
$$

para o caso discreto, e

$$
C^{-1}(\theta)=\int_{S} e^{Q(\theta) T(x)} h(x) d x
$$

para o caso contínuo. Se $X_{1}, \ldots, X_{n}$ são variáveis aleatórias independentes e identicamente distribuídas com função de densidade $f(x, \theta)$ como acima, então a função de densidade conjunta das $X^{\prime} s$ é dada por

$$
\begin{gathered}
f\left(x_{1}, \ldots, x_{n} ; \theta\right)=C^{m}(\theta) \exp \left[Q(\theta) \sum_{j=1}^{n} T\left(x_{j}\right)\right] h\left(x_{1}\right), \ldots, h\left(x_{n}\right), \\
x_{j} \in \mathcal{R}, \quad j=1, \ldots, n, \quad \theta \in \Omega .
\end{gathered}
$$

Algumas generalizações multiparamétricas podem ser feitas, como segue: sejam $X_{1}, \ldots, X_{n}$ variáveis aleatórias independentes e identicamente distribuídas e seja $\mathbf{X}=\left(X_{1}, \ldots, X_{n}\right)^{\prime}$. Diz-se que a função de densidade conjunta das $X^{\prime}$ s, ou que a função de distribuição de $\mathbf{X}$, pertence à família exponencial $r$ paramétrica se:

$$
f(\mathbf{x} ; \boldsymbol{\theta})=C(\boldsymbol{\theta}) \exp \left[\sum_{j=1}^{r} Q_{j}(\boldsymbol{\theta}) T_{j}(\mathbf{x})\right] h(\mathbf{x}),
$$

onde $\mathbf{x}=\left(x_{1}, \ldots, x_{n}\right)^{\prime}, x_{j} \in \mathcal{R}, j=1, \ldots, k, k \geq 1, \boldsymbol{\theta}=\left(\theta_{1}, \ldots, \theta_{\tau}\right)^{\prime} \in \Omega \subseteq R^{r}$, $C(\theta)>0, \boldsymbol{\theta} \in \Omega$ e $h(\mathbf{x})>0$ para $\mathbf{x} \in S$, o conjunto de positividade de $f(x, \theta)$, que é independente de $\boldsymbol{\theta}$. 


\subsection{Modelos Lineares Generalizados}

A idéia de Modelos Lineares Generalizados surgiu quando Nelder e Wedderburn (1972) relataram uma técnica de regressão linear ponderada que é usada para obter os estimadores de máxima verossimilhança dos parâmetros com observações distribuídas de acordo com alguma família exponencial, e efeitos sistemáticos que podem tornar-se linear com uma transformação adequada. Uma generalização da análise da variância é dada para esses modelos usando logaritmos das verossimilhanças. Estes modelos lineares generalizados são ilustrados por quatro distribuições: a normal, binomial (análise probit), poisson (tabelas de contingência) e gama (componentes de variância).

Uma extensão maior da aplicabilidade de Modelos Lineares Generalizados foi feita por Wedderburn (1974) quando ele introduziu a idéia de quaseverossimilhança. O autor mostrou que muitas vezes não é necessário se fazerem suposições específicas detalhadas em relação à variação aleatória. Apesar disso, muitas das propriedades mais utilizadas das estimativas de parâmetros, que resultaram inicialmente da teoria da verossimilhança, podem ser justificadas fundamentando-se em suposições enfraquecidas sobre independência e primeiro e segundo momentos somente. Especificamente, é necessário conhecer como a variância de cada observação muda com seu valor médio mas não é necessário especificar a distribuição em sua totalidade.

Antes de descrever a forma de um modelo linear generalizado, primeiramente serão definidas as etapas de ajuste de um modelo, segundo McCullagh e Nelder (1991):

i) Seleção do Modelo

ii) Estimação dos parâmetros

iii) Predição de valores futuros. 
Veja agora a definição de cada um desses três passos:

\subsubsection{Seleção do Modelo}

O modelo selecionado para se ajustar dados deve ser escolhido de uma classe particular e, se o processo de ajuste do modelo é utilizado, esta classe deve ser relevante ao tipo de dados sob estudo. Em modelos lineares generalizados, há uma característica importante que é a suposição de independência dos erros. Como consequência disso, dados exibindo autocorrelações de séries temporais e processos espaciais são excluídos. A suposição de independência é característica de um modelo linear da análise de regressão clássica, sendo levada sem modificações para uma classe mais ampla de modelos lineares generalizados.

A escolha de escala para a análise é um importante aspecto da seleção do modelo. A escolha mais comum é entre a análise de $Y$, isto é, a escala original ou o $\ln Y$. Para a pergunta "O que caracteriza uma boa escala?" deve-se responder que tudo depende do propósito para o qual a escala está sendo usada. Numa análise de regressão linear clássica, uma boa escala deve combinar homogeneidade de variâncias, normalidade aproximada dos erros e aditividade dos efeitos sistemáticos. No caso de análise de dados discretos onde os resíduos são aproximados pela distribuição de Poisson, os efeitos sistemáticos são, na maioria das vezes, multiplicativos. Aqui,

$Y^{\frac{1}{2}}$ dá uma melhora da homogeneidade das variâncias, $Y^{\frac{2}{3}}$ melhora a simetria ou normalidade, e $\ln Y$ produz a aditividade dos efeitos sistemáticos.

Com a introdução dos modelos lineares generalizados, problemas com escala são grandemente reduzidos, pois utilizando-se este enfoque, não se exigem as pressuposições de homogeneidade de variâncias, normalidade dos erros e aditividade dos efeitos sistemáticos.

Assim, caso possa ser definido exatamente o significado de um modelo ótimo num dado contexto, é mais improvável que os dados indicariam um melhor entre o grande número de modelos que estão entre os escolhidos. 


\subsubsection{Estimação}

Tendo escolhido um modelo particular, é necessário estimar os parâmetros e conhecer a precisão dos estimadores. No caso de modelos lineares generalizados, a estimação processa-se definindo uma medida de qualidade do ajuste entre os dados observados e os valores gerados pelo modelo. Os estimadores dos parâmetros são os valores que minimizam o critério de ajuste.

Tem-se a seguir os estimadores obtidos pela maximização da verossimilhança ou do logaritmo da função de verossimilhança dos parâmetros para os dados observados.

Se $f(y ; \theta)$ é a função de densidade ou distribuição de probabilidade para a observação $y$ dado o parâmetro $\theta$, então o logaritmo da função de verossimilhança, expressa comò uma função do valor médio do parâmetro, $\mu=E(Y)$, é

$$
l(\mu ; y)=\ln f(y ; \theta)
$$

O logaritmo da função de verossimilhança baseado num conjunto de observações independentes $y_{1}, \ldots, y_{n}$ é a soma de contribuições individuais, tais que

$$
l(\boldsymbol{\mu} ; \mathbf{y})=\sum_{i} \ln f_{i}\left(y_{i} ; \theta_{i}\right)
$$

em que $\boldsymbol{\mu}=\left(\mu_{1}, \ldots, \mu_{n}\right)$. Note que a função de densidade $f(y ; \theta)$ é considerada como uma função de $y$ para $\theta$ fixo, enquanto que o logaritmo da função de verossimilhança é primeiramente considerado como uma função de $\theta$ para os $y$ 's observados.

\subsubsection{Predição}

Predição está concentrada em informações sobre valores parecidos de eventos não observados, não necessariamente aqueles no futuro. Para utilizar isso, têm-se que quantidades previsivas precisam ser acompanhadas por medidas de precisão. 
Há a suposição de que o conjunto de dados permanece constante, e o modelo usado na análise deve ser substancialmente correto.

\subsubsection{Os componentes de um Modelo Linear Generalizado}

Segundo McCullagh e Nelder (1991), um vetor de observações y com $n$ componentes é suposto ser uma realização de uma variável aleatória $\mathrm{Y}$ cujos componentes são independentemente distribuídos com média $\boldsymbol{\mu}$. No caso de modelos lineares ordinários, esta média toma a forma

$$
\boldsymbol{\mu}=\sum_{j=1}^{p} \mathbf{x}_{j} \beta_{j}
$$

em que os $\beta$ 's são parâmetros cujos valores são usualmente desconhecidos e são estimados através dos dados e os $x_{j}$ são variáveis explanatórias. Se for tomado $i$ como índice das observações, então, a parte sistemática do modelo deve ser escrita

$$
E\left(Y_{i}\right)=\mu_{i}=\sum_{j=1}^{p} x_{i j} \beta_{j} ; \quad i=1, \ldots, n
$$

em que $x_{i j}$ é o valor da $j$-ésima covariável para a observação $i$. Em notação matricial (em que $\boldsymbol{\mu}$ é um vetor $n \times 1, \mathrm{X}$ é uma matriz $n \times p$ e $\boldsymbol{\beta}$ é um vetor $p \times 1$ ) escreve-se

$$
\boldsymbol{\mu}=\mathbf{X} \boldsymbol{\beta}
$$

considerando $\mathbf{X}$ como a matriz do modelo e $\boldsymbol{\beta}$ o vetor de parâmetros. Isto completa a parte sistemática do modelo.

Para a parte aleatória, supõe-se a independência e variâncias constantes dos resíduos. Estas suposições são fortes e precisam ser muito bem checadas, utilizando-se os dados. Do mesmo modo, a estrutura da parte sistemática supõe que se conhecem as covariáveis que influem na média e pode-se medí-las efetivamente sem erros. 
Uma posterior adaptação do modelo envolve a mais forte suposição de que os resíduos seguem uma distribuição normal ou Gaussiana com variância constante $\sigma^{2}$.

Pode-se, assim, resumir o modelo linear clássico da seguinte forma: os componentes de $\mathrm{Y}$ são variáveis normais independentes com variância constante $\sigma^{2}$ e

$$
E(\mathbf{Y})=\boldsymbol{\mu} \quad \text { onde } \quad \boldsymbol{\mu}=\mathbf{X} \boldsymbol{\beta}
$$

Ampliando esta idéia para modelos lineares generalizados, deve-se fazer uma nova especificação composta de três partes:

1. O componente aleatório: os componentes de $\mathbf{Y}$ têm distribuições Normais independentes com $E(\mathbf{Y})=\boldsymbol{\mu}$ e variância constante $\sigma^{2}$;

2. O componente sistemático: covariáveis $\mathbf{x}_{1}, \mathbf{x}_{2}, \ldots, \mathbf{x}_{p}$ produzem um preditor linear $\boldsymbol{\eta}$ dado por

$$
\boldsymbol{\eta}=\sum_{j=1}^{p} \mathbf{x}_{j} \beta_{j}
$$

3. A ligação entre os componentes aleatório e sistemático:

$$
\boldsymbol{\mu}=\boldsymbol{\eta}
$$

Esta generalização introduz um novo símbolo $\eta$ para o preditor linear e o terceiro componente então especifica que $\mu$ e $\eta$ são de fato idênticos. Se é escrito:

$$
\eta_{i}=g\left(\mu_{i}\right)
$$

então $g($.$) será chamada de função de ligação. Dentro desta formulação, modelos$ lineares clássicos tem uma distribuição normal (ou Gaussiana) no componente (1) e a função identidade para a ligação no componente (3). Modelos lineares generalizados 
permitem três extensões; primeiro, a distribuição no componente (1) pode vir de um outro membro da família exponencial que não a normal, e não é necessário ter variância constante; segundo, a função de ligação no componente (3) pode tornarse qualquer função monotônica diferenciável e, terceiro, a variância não precisa ser constante na especificação (1) quando a distribuição não é a normal.

Supõe-se agora que cada componente de $\mathbf{Y}$ tem uma distribuição na família exponencial, tomando a forma

$$
f_{Y}(y ; \theta, \phi)=\exp \{(y \theta-b(\theta)) / a(\phi)+c(y, \phi)\}
$$

para algumas funções específicas $a(),. b($.$) e c($.$) . Se \phi$ é conhecido, este é um modelo de família exponencial com parâmetro canônico $\theta$. Ela pode ser, ou não, uma família exponencial biparametrizada se $\phi$ é desconhecido. Assim, para a distribuição normal

$$
\begin{aligned}
f_{Y}(y ; \theta, \phi) & =\frac{1}{\sqrt{\left(2 \pi \sigma^{2}\right)}} \exp \left\{-(y-\mu)^{2} / 2 \sigma^{2}\right\} \\
& =\exp \left\{\left(y \mu-\mu^{2} / 2\right) / \sigma^{2}-\frac{1}{2}\left(y^{2} / \sigma^{2}+\ln \left(2 \pi \sigma^{2}\right)\right)\right\},
\end{aligned}
$$

tem-se que $\theta=\mu, \quad \phi=\sigma^{2}$, e

$$
a(\phi)=\phi, \quad b(\theta)=\theta^{2} / 2, \quad c(y, \phi)=-\frac{1}{2}\left\{y^{2} / \phi+\ln (2 \pi \phi)\right\}
$$

Escreve-se $l(\theta, \phi ; y)=\ln f_{Y}(y ; \theta, \phi)$ para a função de verossimilhança considerada como uma função de $\theta$ e $\phi, y$ sendo dado. A média e variância de $Y$ podem ser obtidas facilmente das conhecidas relações

$$
E\left(\frac{\partial l}{\partial \theta}\right)=0
$$

e

$$
E\left(\frac{\partial^{2} l}{\partial \theta^{2}}\right)+E\left(\frac{\partial l}{\partial \theta}\right)^{2}=0
$$


De ( 8) tem-se que

$$
l(\theta, y)=\{y \theta-b(\theta)\} / a(\phi)+c(y, \phi),
$$

que resulta em

$$
\frac{\partial l}{\partial \theta}=\left\{y-b^{\prime}(\theta)\right\} / a(\phi)
$$

$\mathrm{e}$

$$
\frac{\partial^{2} l}{\partial \theta^{2}}=-b^{\prime \prime}(\theta) / a(\phi),
$$

em que $b^{\prime}($.$) e b^{\prime \prime}($.$) denotam a diferenciação com relação a \theta$.

De (9) e (11) tem-se

$$
0=E\left(\frac{\partial l}{\partial \theta}\right)=\left\{\mu-b^{\prime}(\theta)\right\} / a(\phi),
$$

que fornece

$$
E(Y)=\mu=b^{\prime}(\theta) .
$$

Do mesmo modo, de (10), (11) e (12), tem-se

$$
0=-\frac{b^{\prime \prime}(\theta)}{a(\phi)}+\frac{\operatorname{var}(Y)}{a^{2}(\phi)}
$$

que resulta em

$$
\operatorname{var}(Y)=b^{\prime \prime}(\theta) a(\phi)
$$

Assim, a variância de $Y$ é o produto de duas funções; uma, $b^{\prime \prime}(\theta)$, que depende do parâmetro canônico somente (e também da média) e será chamada de função de variância, enquanto que a outra é independente de $\theta$ e depende somente de $\phi$. A função de variância considerada como uma função de $\mu$ será escrita por $V(\mu)$.

A função $a(\phi)$ é comumente da forma 


$$
a(\phi)=\phi / w
$$

onde $\phi$, também denotado por $\sigma^{2}$ e chamado de parâmetro de dispersão, é constante sobre as observações, e $w$ é um peso a priori conhecido que varia de observação para observação. Assim, para um modelo normal, no qual cada observação é a média de $m$ leituras independentes, tem-se

$$
a(\phi)=\sigma^{2} / m,
$$

então, $w=m$.

A função de ligação relaciona o preditor linear $\eta$ ao valor esperado $\mu$ de uma variável $Y$. Em modelos lineares clássicos a média e o preditor linear são idênticos, e a ligação identidade é plausível em que ambos $\eta$ e $\mu$ podem tomar qualquer valor numa reta real. No entanto, quando se lida com contagens e a distribuição é Poisson, deve-se ter $\mu>0$, então a ligação identidade é menos atrativa, em parte por causa de $\eta$ poder ser negativo enquanto $\mu$ não pode ser. Modelos para contagens baseados na independência de dados em classificação cruzada levam naturalmente a efeitos multiplicativos, e isso é expresso pela ligação logarítmica, $\eta=\ln \mu$, com sua inversa $\mu=e^{\eta}$. Agora efeitos aditivos contribuindo para $\eta$ tornam-se efeitos multiplicativos, contribuindo para $\mu$, e $\mu$ é necessariamente positivo.

Para a distribuição binomial tem-se $0<\mu<1$ e uma ligação deve satisfazer à condição que para o intervalo $(0,1)$, tal função assume valores em toda a reta real. Serão citadas aqui três importantes funções:

\section{1. $\operatorname{logit}$}

$$
\eta=\ln \{\mu /(1-\mu)\}
$$




$$
\eta=\Phi^{-1}(\mu)
$$

onde $\Phi($.$) é a função de distribuição acumulada normal;$

3. complemento log-log

$$
\eta=\ln \{-\ln (1-\mu)\}
$$

\subsection{Medindo a qualidade do ajuste}

O processo para ajustar um modelo aos dados pode ser entendido como um meio de trocar valores de um conjunto de dados y por um conjunto de valores ajustados $\hat{\boldsymbol{\mu}}$ oriundos de um modelo envolvendo normalmente um pequeno número de parâmetros. Em geral os $\boldsymbol{\mu}$ 's não serão iguais aos y's exatamente, e a questão então surge de quão discrepante eles são, já que uma pequena discrepância pode ser tolerável. Medidas de discrepância ou de qualidade do ajuste podem ser fornecidas de vários modos, mas este trabalho vai se concentrar somente naquela formada pelo logaritmo da razão de verossimilhanças, chamada deviance.

Dadas $n$ observações, podem-se ajustar modelos com $n$ parâmetros. O modelo mais simples é chamado modelo nulo, tem um parâmetro comum $\mu$ para todos os $y$ 's; o modelo nulo concentra toda a variação dos $y$ 's no componente aleatório. Em outro extremo, o modelo completo tem $n$ parâmetros, um por observação, e os $\mu$ 's obtidos representam os dados exatamente. O modelo completo concentra, então, toda a variação dos $y$ 's ao componente sistemático não sobrando nenhuma para o componente aleatório. Na prática o modelo nulo é simples demais e o modelo completo é não-informativo, porque ele não representa os dados, apenas os repete. No entanto, o modelo completo dá-nos uma base para se medir a discrepância para um modelo intermediário com $p$ parâmetros. 
Assim, é conveniente se expressar o logaritmo da função de verossimilhança em termos do parâmetro do valor médio $\boldsymbol{\mu}$ do que o parâmetro canônico $\boldsymbol{\theta}$. Seja $l(\hat{\boldsymbol{\mu}}, \phi, \mathbf{y})$ o logaritmo da função de verossimilhança maximizado sobre $\boldsymbol{\beta}$ para um valor fixo do parâmetro de dispersão $\phi$. $O$ logaritmo da função de verossimilhança num modelo completo com $n$ parâmetros é $l(\mathbf{y}, \phi, \mathbf{y})$, que é comumente finito. A deviance de um ajuste é proporcional a duas vezes a diferença entre o logaritmo da função de verossimilhança do modelo completo e a do modelo sob pesquisa. Se forem denotados por $\hat{\boldsymbol{\theta}}=\boldsymbol{\theta}(\hat{\boldsymbol{\mu}})$ e $\tilde{\boldsymbol{\theta}}=\boldsymbol{\theta}(\mathbf{y})$ as estimativas dos parâmetros canônicos sob os dois modelos, a deviance escalonada, supondo $a_{i}(\phi)=\phi / w_{i}$, pode ser escrita

$$
\sum 2 w_{i}\left\{y_{i}\left(\tilde{\theta}_{i}-\hat{\theta}_{i}\right)-b\left(\tilde{\theta}_{i}\right)+b\left(\hat{\theta}_{i}\right)\right\} / \phi=D(\mathbf{y} ; \hat{\boldsymbol{\mu}}) / \phi
$$

onde $D(\mathbf{y} ; \hat{\boldsymbol{\mu}})$ é conhecida como a deviance para o modelo corrente e é uma função somente dos dados.

Existem vantagens em usar como critério de qualidade de ajuste, não o logaritmo da função de verossimilhança $l(\mu ; \mathbf{y})$ mas uma função linear particular, i.é,

$$
D^{*}(\mathbf{y} ; \boldsymbol{\mu})=2 l(\mathbf{y} ; \mathbf{y})-2 l(\boldsymbol{\mu} ; \mathbf{y})
$$

que é chamada deviance escalonada. Note que, para os modelos da família exponencial, $l(\mathbf{y} ; \mathbf{y})$ é a máxima verossimilhança calculada para um ajuste exato no qual os valores ajustados são iguais aos dados observados. Por causa da $l(\mathbf{y} ; \mathbf{y})$ não depender dos parâmetros, maximizar $l(\boldsymbol{\mu} ; \mathbf{y})$ é equivalente a minimizar a $D^{*}(\mathbf{y} ; \boldsymbol{\mu})$ com relação a $\boldsymbol{\mu}$, sujeito às restrições impostas pelo modelo.

A distribuição da deviance é desconhecida, mas sabe-se que, para a distribuição binomial, sua distribuição é assintoticamente $\chi^{2}$ com ordem de convergência $O\left(m^{-3 / 2}\right)$. Para se melhorar essa aproximação da distribuição da deviance, alguns autores propõem alguns fatores de correção, mas para tamanhos de amostra pequenos, essas correções não resolvem o problema. Vejamos quais os fatores de correção propostos: 


\subsection{Correção de Bartlett}

McCullagh e Nelder (1991) apresentam a seguinte proposta: uma hipótese nula simples $H_{0}: \boldsymbol{\theta}=\boldsymbol{\theta}_{0}$ pode ser testada usando a estatística da razão de verossimilhanças, que é dada por duas vezes a diferença entre o logaritmo da função de verossimilhança em $\boldsymbol{\theta}$ e o valor obtido em $\boldsymbol{\theta}_{\mathbf{0}}$. Para modelos lineares generalizados nos quais o parâmetro de dispersão é conhecido, esta diferença pode ser escrita em termos das deviances como segue:

$$
\Lambda=2 l(\hat{\boldsymbol{\theta}} ; Y)-2 l\left(\boldsymbol{\theta}_{0} ; Y\right)=D\left(Y ; \boldsymbol{\theta}_{0}\right)-D(Y ; \hat{\boldsymbol{\theta}}) .
$$

Supõe-se aqui, para simplificar a notação, que o parâmetro de dispersão é igual à unidade.

Sob as condições usuais de regularidade assintótica para grandes amostras a média assintótica desta estatística é

$$
\begin{aligned}
E\left\{D\left(Y, \boldsymbol{\theta}_{0}\right)-D(Y ; \hat{\boldsymbol{\theta}})\right\} & =p+\epsilon_{p}+O\left(n^{-2}\right), \\
& =p\left\{1+b_{p}\left(\boldsymbol{\theta}_{0}\right)\right\}+O\left(n^{-2}\right),
\end{aligned}
$$

onde $p=\operatorname{dim}(\boldsymbol{\theta})$ e $b(\boldsymbol{\theta})$ é conhecido como o fator de Correção de Bartlett. O cumulante de ordem $r$ é dado por:

$$
\kappa_{r}\left\{D\left(Y ; \boldsymbol{\theta}_{0}\right)-D(Y ; \hat{\boldsymbol{\theta}})\right\}=(r-1) ! 2^{r-1} p\left\{1+b_{p}\left(\boldsymbol{\theta}_{0}\right)\right\}^{r}+O\left(n^{-2}\right)
$$

O principal termo nesta expressão é o $r$-ésimo cumulante da distribuição de $\chi_{p}^{2}$. Da propriedade multiplicativa de cumulantes pode ser visto imediatamente que os cumulantes da estatística ajustada

$$
\Lambda^{\prime}=\frac{\Lambda}{1+b_{p}}
$$


são os mesmos da distribuição de $\chi_{p}^{2}$, quando termos de ordem $O\left(n^{-2}\right)$ são desprezados. Tem-se que $b_{p}$ e $\epsilon_{p}$ são ambos $O\left(n^{-1}\right)$, por suposição.

Apesar da convergência de os cumulantes implicar na convergência à distribuição requerida, a ordem de magnitude da discrepância neles não é necessariamente do mesmo tamanho do erro na função de distribuição acumulada. Contudo, parece razoável concluir que a distribuição da estatística ajustada é dada por

$$
\Lambda^{\prime} \sim \chi_{p}^{2}+O\left(n^{-2}\right)
$$

Para hipóteses nulas compostas, que são mais comuns em aplicações, uma correção similar pode ser feita. A estatística pode ser escrita da forma

$$
\Lambda\left(\hat{\boldsymbol{\theta}}, \hat{\boldsymbol{\theta}}_{0}\right)=2 l(\hat{\boldsymbol{\theta}} ; Y)-2 l\left(\hat{\boldsymbol{\theta}}_{0} ; Y\right)=D\left(Y ; \hat{\boldsymbol{\theta}}_{0}\right)-D(Y ; \hat{\boldsymbol{\theta}}),
$$

onde $\hat{\boldsymbol{\theta}}_{0}$ é a estimativa dos parâmetros nuisance sob $H_{0}$, e $\hat{\boldsymbol{\theta}}$ é a estimativa irrestrita. Supondo que as hipóteses são aninhadas, e que $q<p$ é a dimensão do espaço paramétrico sob $H_{0}$, a média desta estatística é

$$
\begin{aligned}
E\left\{\Lambda\left(\hat{\boldsymbol{\theta}}, \hat{\boldsymbol{\theta}}_{0}\right)\right\} & =p+\epsilon_{p}+O\left(n^{-2}\right)-\left(q+\epsilon_{q}+O\left(n^{-2}\right)\right) \\
& =p-q+\left(\epsilon_{p}-\epsilon_{q}\right)+O\left(n^{-2}\right) \\
& =(p-q)\left\{1+b_{p q}(\boldsymbol{\theta})\right\}+O\left(n^{-2}\right) .
\end{aligned}
$$

Assim o fator de correção requerido é agora

$$
b_{p q}=\left(p b_{p}-q b_{q}\right) /(p-q)=\left(\epsilon_{p}-\epsilon_{q}\right) /(p-q) .
$$

Os cumulantes da estatística da razão de verossimilhanças (14) obedecem a (13), com $p$ substituído por $p-q$. Assim os cumulantes da estatística corrigida continuam os mesmos da distribuição de $\chi_{p-q}^{2}$, separados dos termos de ordem $O\left(n^{-2}\right)$. 


\subsection{Propostas de Cordeiro (1986)}

Cordeiro (1986) relata que para examinar propriedades da deviance em pequenas amostras, torna-se necessária uma aproximação de ordem superior para o seu valor esperado, supondo o modelo sob pesquisa verdadeiro. Tem-se que a definição da deviance é $D_{p}=2\left(\hat{L}_{n}-L\right)+2\left(L-\hat{L}_{p}\right)$, onde $\hat{L}_{n}, \hat{L}_{p}$ e $L$ são os valores de $L(\beta)$, dados a seguir, nos pontos $y, \hat{\mu}$ e no parâmetro verdadeiro, respectivamente:

$$
L(\beta)=\sum_{l=1}^{n} \phi_{l}\left[y_{l} \theta_{l}-b\left(\theta_{l}\right)+c\left(y_{l}, \phi_{l}\right)\right]
$$

onde $\theta_{l}=q\left(\mu_{l}\right), \mu_{l}=f^{-1}(\eta), \eta=\sum_{i=1}^{p} x_{l i} \beta_{i}$ e $\phi_{l}$ é suposto conhecido para cada observação.

Daí, o valor esperado da deviance é expresso, até termos de ordem $n^{-1}$, por

$$
E\left(D_{p}\right)=2 E\left(\hat{L}_{n}-L\right)-\left(p+\epsilon_{p}\right)+O\left(n^{-2}\right)
$$

O termo $2\left(\hat{L}_{n}-L\right)$ representa a deviance no parâmetro verdadeiro e o seu valor esperado pode ser calculado a partir da distribuição proposta para os dados. Dado que:

$$
\hat{L}_{n}=\sum_{l=1}^{n} \phi_{l}\left[y_{l} q\left(y_{l}\right)-b\left(q\left(y_{l}\right)\right)+c\left(y_{l}, \phi_{l}\right)\right]
$$

vem, até ordem $n^{-1}$,

$$
E\left(D_{p}\right)=2 \sum_{l=1}^{n} \phi_{l}\left\{E\left[v\left(Y_{l}\right)\right]-v\left(\mu_{l}\right)\right\}-\left(p+\epsilon_{p}\right)
$$

em que

$$
v(x)=x q(x)-b(q(x))
$$

$\operatorname{com} b($.$) sendo uma função conhecida e q()=.\theta$. 
Todos os termos em (16) devem ser calculados de acordo com o modelo sob pesquisa.

O termo de ordem $n$ em (16) é uma função das médias verdadeiras $\mathrm{e}$ desconhecidas, para o Modelo Binomial. Este termo pode ser calculado exatamente e a aproximação assintótica considerada é somente quando $n$ tende a infinito.

A vantagem de (16) é que se pode deduzir um fator de correção:

$$
c=(n-p) / E\left(D_{p}\right)
$$

Daí, pode-se definir uma deviance modificada $D_{p}^{*}=c D_{p}$ para o modelo sob pesquisa, tal que $E\left(D_{p}^{*}\right)$ é melhor aproximada por $(n-p)$ do que $E\left(D_{p}\right)$. Isto não implica, necessariamente, que $D_{p}^{*}$ é melhor aproximada por uma distribição $\chi_{n-p}^{2}$.

Frequentemente, $E\left(D_{p}\right)$ é uma função das médias desconhecidas e estas são estimadas a partir de $\tilde{\boldsymbol{\beta}}=\left(\tilde{\beta}_{1} \ldots \tilde{\beta}_{p}\right)^{T}$ para calcular um valor numérico para c. Isto claramente não afetará a ordem da aproximação obtida. Pelo menos, com grandes amostras, os momentos de $D_{p}^{*}$ devem ser mais próximos dos correspondentes momentos da distribuição $\chi_{n-p}^{2}$ do que aqueles de $D_{p}$. Mas, para $n$ pequeno, isto, em geral, não é verdadeiro.

Gart e Zweifel (1967) sugerem a adição de 0, 5 às freqüências observadas para aperfeiçoar a aproximação do $\chi^{2}$ de referência. Essa aproximação da distribuição da deviance por uma distribuição $\chi^{2}$ será bastante razoável, quando todos os $\mu_{l}$ 's forem maiores do que 5 .

\subsection{Proposta de Cox e Snell (1968) para Transformação da Variável Aleatória}

Para aplicar os métodos de se calcularem resíduos para dados com distribuição binomial, deve-se primeiro considerar como definir resíduos e como obter variáveis identicamente distribuídas aproximadas. Aqui, não será definido o modelo diretamente, mas sim proceder-se-á indiretamente, definindo $R_{i}$ como: 
a) $\left(Y_{i}-\mu_{i}\right) / \sqrt{\mu_{i}}$,

b) $2\left(\sqrt{Y_{i}}-\sqrt{\mu_{i}}\right)$, ou

c) $\left\{\psi\left(Y_{i}\right)-\psi\left(\mu_{i}\right)\right\} /\left\{\psi^{\prime}\left(\mu_{i}\right) \sqrt{\mu_{i}}\right\}$

onde $\mu_{i}=\mu_{i}(\hat{\boldsymbol{\beta}})$ é a freqüência esperada, de algum modelo dependente sob os parâmetros $\beta_{1}, \ldots, \beta_{p}$ da observação binomial, $Y_{p}$. Cada um deles, assintoticamente, define um desvio normal padronizado, (a) e (b) surgem do Teorema de Slutsky para convergência de variáveis aleatórias; (c) é uma generalização de $(a)$ e $(b)$, e no qual $\psi(x)$ é uma função arbitrária.

A escolha de uma transformação apropriada depende das exigências; a necessidade para uma interpretação direta deve levar a $(a)$ ou, alternativamente, para um modelo multiplicativo, por exemplo, $(c) \operatorname{com} \psi(x)=\ln x$. Desde que o objetivo imediato é obter os resultados das análises dos dados melhor do que explicá-los, é desejável encontrar uma transformação que resulte num conjunto de resíduos com uma distribuição mais próxima possível de alguma forma conhecida.

Seguindo os argumentos anteriores, define-se uma transformação $\phi\left(Y_{i} / m_{i}\right)$ da observação $Y_{i}$ de uma distribuição binomial com parâmetros $\theta_{i}(\boldsymbol{\beta})$ e $m_{i}$. Considerando a expansão de Taylor e igualando a assimetria a zero, obtém-se uma equação diferencial cuja solução é

$$
\phi(\mu)=\int_{0}^{\mu} t^{-\frac{1}{3}}(1-t)^{-\frac{1}{3}} d t, \quad 0 \leq \mu \leq 1 .
$$

Blom (1954) sugere a equação ( 17) como uma transformação normalizadora, mas não a aplica. De maneira a simplificar sua aplicação, foi calculada a tabela da função beta. Ela dá os valores de $\phi(\mu) / \phi(1)$, isto é, a função beta incompleta $I_{\mu}\left(\frac{2}{3}, \frac{2}{3}\right)$, que é simétrica em torno de $\mu=0,5$; a multiplicação por $B\left(\frac{2}{3}, \frac{2}{3}\right)=2,0533$ dá o valor de ( 17). Por exemplo, $\phi(0,2)=2,0533 \times 0,257=0,528, \quad \phi(0,8)=$ $2,0533(1-0,257)=1,526$. 
Introduzindo a média e a variância da variável binomial transformada, pode-se definir

$$
h_{i}\left(Y_{i}, \boldsymbol{\beta}\right)=\left[\phi\left(Y_{i} / m_{i}\right)-\phi\left\{\theta_{i}-\frac{1}{6}\left(1-2 \theta_{i}\right) / m_{i}\right\}\right] /\left\{\theta_{i}^{\frac{1}{6}}\left(1-\theta_{i}\right)^{\frac{1}{6}} / \sqrt{m_{i}}\right\}
$$

que se reduz a

$$
\left\{Y_{i}^{\frac{2}{3}}-\left(\mu_{i}-\frac{1}{6}\right)^{\frac{2}{3}}\right\} /\left(\frac{2}{3} \mu_{i}^{\frac{1}{6}}\right)
$$

para $\theta_{i}$ pequeno. Gráficos do artigo sugerem que a transformação é muito efetiva, mesmo para valores pequenos de $m_{i}$ e $\theta_{i}$. Muitas vezes a correção do viés, dada por $-\frac{1}{6}\left(1-2 \theta_{i}\right) / m_{i}$ pode ser omitida.

\subsection{Desenvolvimentos Assintóticos - Série de Taylor}

Kaplan (1973) dá a seguinte definição de série de Taylor: Seja $f(x)$ a soma de séries ascendentes com intervalo de convergência $a-r^{*}<x<a+r^{*}\left(r^{*}>0\right)$ :

$$
f(x)=\sum_{n=0}^{\infty} c_{n}(x-a)^{n}, \quad a-r^{*}<x<a+r^{*} .
$$

Esta série é chamada de série de Taylor de $f(x)$ para $x=a$ se os coeficientes $c_{n}$ são dados pela regra:

$$
c_{0}=f(a), \quad c_{1}=\frac{f^{\prime}(a)}{1 !}, \quad c_{2}=\frac{f^{\prime \prime}(a)}{2 !}, \ldots, \quad c_{n}=\frac{f^{(n)}(a)}{n !}, \ldots
$$

tais que

$$
f(x)=f(a)+\frac{f^{\prime}(a)}{1 !}(x-a)+\ldots+\frac{f^{(n)}(a)}{n !}(x-a)^{n}+\ldots
$$

Teorema. Todas as séries ascendentes com convergência de área não nula são a série de Taylor desta soma. 
Prova. Seja $f(x)$ dada por ( 19). Então, por repetidas diferenciações, encontra-se que:

$$
\begin{gathered}
f(x)=c_{0}+c_{1}(x-a)+\ldots+c_{n}(x-a)^{n}+\ldots, \\
f^{\prime}(x)=c_{1}+2 c_{2}(x-a)+\ldots+n \cdot c_{n}(x-a)^{n-1}+\ldots, \\
f^{\prime \prime}(x)=2 c_{2}+6 c_{3}(x-a)+\ldots+n(n-1) \cdot c_{n}(x-a)^{n-2}+\ldots, \\
\ldots \ldots, \\
f^{(n)}(x)=n(n-1)(n-2) \ldots 2.1 \cdot c_{n} \\
+(n+1) n(n-1) \ldots 2 \cdot c_{n+1}(x-a)+\ldots,
\end{gathered}
$$

Aqui todas as séries convergem para $a-r^{*}<x<a+r^{*}$. Fazendo agora $x=a$, segue que

$$
f(a)=c_{0}, \quad f^{\prime}(a)=c_{1}, \quad f^{\prime \prime}(a)=2 c_{2}, \ldots, \quad f^{(n)}(a)=n ! c_{n}, \ldots
$$

Assim, $c_{0}=f(a) \mathrm{e}$

$$
c_{n}=\frac{f^{(n)}(a)}{n !}, \quad n=1,2, \ldots
$$

encerrando a prova $\diamond$.

No caso de $a=0$, a expressão ( 20) para a série de Taylor de $f(x)$ torna-se

$$
f(x)=f(0)+\frac{f^{\prime}(0)}{1 !} x+\frac{f^{\prime \prime}(0)}{2 !} x^{2}+\ldots+\frac{f^{(n)}(0) x^{n}}{n !}+\ldots
$$

Esta expressão é chamada série de Maclaurin de $f(x)$ que, para muitos propósitos, é mais fácil de se usar. A substituição $t=x-a$ reduz a série de Taylor geral à forma de Maclaurin. 


\subsubsection{Série de Taylor para funções de uma variável}

De acordo com Lang (1976), pela teoria das funções de uma variável, obtém-se a seguinte expressão para os valores de uma função $f$ na vizinhança de um ponto $a$, por meio das derivadas:

$$
f(a+h)=f(a)+f^{\prime}(a) h+\frac{f^{(2)}(a)}{2 !} h^{2}+\ldots+\frac{f^{(r-1)}(a)}{(r-1) !} h^{r-1}+R_{r}
$$

em que

$$
R_{r}=\frac{f^{(r)}(c)}{r !} h^{r}
$$

para algum ponto $c$ entre $a$ e $a+h$.

\subsubsection{Série de Taylor para funções de Duas ou mais Variáveis}

É possível, de acordo com Lang (1976), obter uma função de duas ou mais variáveis expandida em série de Taylor. Seja uma função $f$ de $n$ variáveis definida num conjunto aberto $U$, e tendo derivadas parciais contínuas até a ordem $r$. Sejam $P$ um ponto de $U$, e $H$ um vetor. Suponha que o segmento de reta

$$
P+t H, \quad 0 \leq t \leq 1,
$$

esteja contido em $U$. Então existe um número $\tau$ entre 0 e 1 tal que

$$
\begin{aligned}
f(P+H) & =f(P)+\frac{(H \cdot \nabla) f(P)}{1 !}+\ldots+\frac{(H . \nabla)^{r-1} f(P)}{(r-1) !}+ \\
& +\frac{(H . \nabla)^{r} f(P+\tau H)}{r !} .
\end{aligned}
$$

Esta forma é obtida substituindo na Fórmula de Taylor para uma variável as derivadas da função $g(t)=f(P+t H)$ pelas suas expressões. Tem-se, assim, que

$$
g^{(s)}(0)=(H . \nabla)^{s} f(P)
$$




$$
g^{(r)}(\tau)=(H . \nabla)^{r}(P+\tau H)
$$

Reescrevendo em termos das notações $\partial / \partial x$ e $\partial / \partial y$, segue que

$$
\begin{aligned}
f(a+h, b+k) & =f(a, b)+\left(h \frac{\partial}{\partial x}+k \frac{\partial}{\partial y}\right) f(a, b)+\ldots+ \\
& +\left(h \frac{\partial}{\partial x}+k \frac{\partial}{\partial y}\right)^{r-1} f(a, b)+ \\
& +\left(h \frac{\partial}{\partial x}+k \frac{\partial}{\partial y}\right)^{r} f(a+\tau h, b+\tau k) .
\end{aligned}
$$

As potências dos operadores diferenciais

$$
\left(h \frac{\partial}{\partial x}+k \frac{\partial}{\partial y}\right)
$$

são calculadas pelo desenvolvimento do binômio. Por exemplo:

$$
\begin{gathered}
\left(h \frac{\partial}{\partial x}+k \frac{\partial}{\partial y}\right)^{2}=h^{2} \frac{\partial^{2}}{\partial x^{2}}+2 h k \frac{\partial^{2}}{\partial x \partial y}+k^{2} \frac{\partial^{2}}{\partial y^{2}} \\
\left(h \frac{\partial}{\partial x}+k \frac{\partial}{\partial y}\right)^{3}=h^{3}\left(\frac{\partial}{\partial x}\right)^{3}+3 h^{2} k\left(\frac{\partial}{\partial x}\right)^{2}\left(\frac{\partial}{\partial y}\right)+ \\
+3 h k^{2}\left(\frac{\partial}{\partial x}\right)\left(\frac{\partial}{\partial y}\right)^{2}+k^{3}\left(\frac{\partial}{\partial y}\right)^{3}
\end{gathered}
$$

e assim por diante.

Segundo Kaplan (1973), há uma fórmula de Taylor com resíduo para funções de $n$ variáveis:

$$
\begin{aligned}
F(x, y)= & F\left(x_{1}, y_{1}\right)+d F\left(x_{1}, y_{1} ; x-x_{1}, y-y_{1}\right) \\
& +\ldots+\frac{1}{n !} d^{n} F\left(x_{1}, y_{1} ; x-x_{1}, y-y_{1}\right) \\
& +\frac{1}{(n+1) !} d^{n+1} F\left(x^{*}, y^{*} ; x-x_{1}, y-y_{1}\right)
\end{aligned}
$$


$\operatorname{com} x^{*}=x_{1}+t^{*}\left(x-x_{1}\right), \quad y^{*}=y_{1}+t^{*}\left(y-y_{1}\right), \quad 0<t^{*}<1$.

O ponto $\left(x^{*}, y^{*}\right)$ se encontra entre $\left(x_{1}, y_{1}\right)$ e $(x, y)$ na linha de junção desses pontos. Para $n=1$, a fórmula se torna:

$$
F(x, y)=F\left(x_{1}, y_{1}\right)+\left(x-x_{1}\right) F_{x}\left(x^{*}, y^{*}\right)+\left(y-y_{1}\right) F_{y}\left(x^{*}, y^{*}\right)
$$

que é conhecida como a Lei da Média para funções de duas variáveis.

Para provar (22), pode-se escrever:

$$
\phi(t)=F\left[x_{1}+t\left(x-x_{1}\right), y_{1}+t\left(y-y_{1}\right)\right], \quad 0 \leq t \leq 1
$$

Tem-se que $x$ e $y$ são considerados fixos e $\phi$ depende somente de $t$. Pela lei da média para $\phi$,

$$
\phi(1)=\phi(0)+\phi^{\prime}\left(t^{*}\right), \quad 0<t^{*}<1
$$

$\operatorname{Mas} \phi(1)=F(x, y), \phi(0)=F\left(x_{1}, y_{1}\right) \mathrm{e}$

$$
\begin{aligned}
\phi^{\prime}(t)= & \left(x-x_{1}\right) F_{x}\left[x_{1}+t\left(x-x_{1}\right), y_{1}+t\left(y-y_{1}\right)\right] \\
& +\left(y-y_{1}\right) F_{y}\left[x_{1}+t\left(x-x_{1}\right), y_{1}+t\left(y-y_{1}\right)\right] .
\end{aligned}
$$

Se $t$ for substituído por $t^{*}$, obtém-se (22). A fórmula geral (21) é provada da mesma maneira, baseada na fórmula de Taylor para $\phi$ :

$$
\phi(1)=\phi(0)+\phi^{\prime}(0)+\ldots+\frac{\phi^{(n)}(0)}{n !}+\frac{\phi^{(n+1)}\left(t^{*}\right)}{(n+1) !},
$$

onde $0<t^{*}<1$. Pode-se encontrar, por indução, que

$$
\phi^{(n)}(t)=d^{n} F\left[x_{1}+t\left(x-x_{1}\right), y_{1}+t\left(y-y_{1}\right) ; x-x_{1}, y-y_{1}\right]
$$


e a validade desta equação é assegurada se $F(x, y)$ tem derivadas contínuas através da $(n+1)$-ésima ordem num domínio $D$ contendo o segmento de linha que liga $(x, y)$ a $\left(x_{1}, y_{1}\right)$.

A série de Taylor ou Fórmula de Taylor pode ser usada para estudar a natureza de uma função perto de um ponto particular. Como foi dito anteriormente, os termos lineares resultam em $d F$, a melhor "aproximação linear" para $F(x, y)$ $F\left(x_{1}, y_{1}\right)$. Se $d F=0$, os termos quadráticos $d^{2} F / 2$ ! tornam-se de grande importância. Em particular, se a expressão quadrática

$$
d^{2} F=A\left(x-x_{1}\right)^{2}+2 B\left(x-x_{1}\right)\left(y-y_{1}\right)+C\left(y-y_{1}\right)^{2}
$$

for positiva, exceto para $x=x_{1}, y=y_{1}$, então $F(x, y)$ tem um mínimo no ponto $\left(x_{1}, y_{1}\right)$.

Em estatística, segundo Leite e Singer (1990), uma das ferramentas mais utilizadas no estudo de métodos assintóticos é a aproximação de uma função real $f$ de variável real por um polinômio. Embora a expansão de $f$ em série de potências na vizinhança de um ponto $x_{0}$ seja importante sob o ponto de vista teórico, o que realmente interessa nas aplicações é o estudo do resto proveniente da aproximação de $f$ por um polinômio.

Para uma função expandida em série de Taylor, para todo $x \in U$, $R_{n}(x)=f(x)-P(x)$ é o resto ou o erro da aproximação de $f(x)$ por $P(x)$. Logo, se $f$ for derivável até a ordem $n$ em um ponto $x_{0}$, tem-se:

$$
f(x)=\sum_{k=0}^{n} \frac{f^{(k)}\left(x_{0}\right)}{k !}\left(x-x_{0}\right)^{k}+R_{n}(x),
$$

para todo $x \in U$. A expressão acima é denominada fórmula de Taylor, com resto $R_{n}$, de $f$ em torno de $x_{0}$. Tem-se que, para $n=1$ e $2, R_{n}(x)$ tende a zero mais rapidamente do que $\left(x-x_{0}\right)^{n}$ quando $x$ tende a $x_{0}$.

Teorema. Seja $f$ uma função real de variável real derivável até a ordem $n+1$ em um intervalo $I$ e sejam $x_{0}, x \in I$. Então existe pelo menos um ponto $c$ entre $x_{0}$ e $x$ tal que 


$$
f(x)=\sum_{k=0}^{n} \frac{f^{(k)}\left(x_{0}\right)}{k !}\left(x-x_{0}\right)^{k}+\frac{f^{(n+1)}(c)}{(n+1) !}\left(x-x_{0}\right)^{n+1} .
$$

Em outras palavras, o resto na fórmula de Taylor é dado por

$$
R_{n}(x)=\frac{f^{(n+1)}(c)}{(n+1) !}\left(x-x_{0}\right)^{n+1} .
$$

Observa-se que, quando $n=0$, este teorema é precisamente o teorema do valor médio. (A prova deste teorema pode ser encontrada em Courant, 1958, cap. VI).

Leite e Singer (1990) definem, a seguir, a notação $O($.$) e o($.$) , que é$ muito usada no estudo de métodos assintóticos, quando se comparam sequências de variáveis aleatórias, e que será utilizada na próxima seção: sejam $\left\{a_{n}\right\}_{n \geq 1}$ e $\left\{b_{n}\right\}_{n \geq 1}$ sequências de números reais. Pode-se dizer que:

i) $a_{n}=O\left(b_{n}\right)$ se existirem um número real $K>0$ e um número inteiro positivo $n_{0}=n_{0}(K)$ tal que $\left|a_{n} / b_{n}\right| \leq K, \quad \forall n \geq n_{0} ;$

ii) $a_{n}=o\left(b_{n}\right)$ se para todo $\epsilon>0$ existir um número inteiro positivo $n_{0}=n_{0}(\epsilon)$ tal que $\left|a_{n} / b_{n}\right|<\epsilon, \quad \forall n \geq n_{0}$.

Ou seja, pode-se dizer que $a_{n}=O\left(b_{n}\right)$ se a razão $\left|a_{n} / b_{n}\right|$ for limitada para todo $n$ suficientemente grande e que $a_{n}=o\left(b_{n}\right)$ se $a_{n} / b_{n} \rightarrow 0$ quando $n \rightarrow \infty$. Em particular, $a_{n}=O(1)$ se existir um número real $K>0$ tal que $\left|a_{n}\right| \leq K$ para todo $n$ suficientemente grande e $a_{n}=o(1)$ se $a_{n} \rightarrow 0$ quando $n \rightarrow \infty$. Essencialmente, a definição anterior está relacionada com a comparação das ordens de magnitude (ou de grandeza) de $\left\{a_{n}\right\}_{n \geq 1}$ e $\left\{b_{n}\right\}_{n \geq 1}$. A grosso modo, afirmar que $a_{n}=O\left(b_{n}\right)$ corresponde a dizer que a ordem de magnitude de $\left\{a_{n}\right\}_{n \geq 1}$ é, no máximo, igual à de $\left\{b_{n}\right\}_{n \geq 1}$ para todo $n$ suficientemente grande; do mesmo modo, afirmar que $a_{n}=o\left(b_{n}\right)$ corresponde a dizer que a ordem de magnitude de $\left\{a_{n}\right\}_{n \geq 1}$ é menor do que a de $\left\{b_{n}\right\}_{n \geq 1}$, para todo $n$ suficientemente grande. 
Em Leite e Singer (1990), ainda, pode-se encontrar uma série de aplicações sobre o uso das expansões em Taylor e Maclaurin em estatística, como, por exemplo, a Lei dos Grandes Números e o Teorema Central do Limite. 


\section{METODOLOGIA}

\subsection{A deviance para a distribuição binomial}

A qualidade de um ajuste em Modelos Lineares Generalizados é medida pela discrepância entre o logaritmo da função de verossimilhança do modelo completo e o do modelo sob pesquisa. Esta medida é conhecida como deviance.

Dados de proporções provêm quase sempre de uma distribuição binomial. Assim, se $Y \sim b(m, \pi)$, sua distribuição de probabilidade é dada por

$$
p(y, \pi)=\left(\begin{array}{c}
m \\
y
\end{array}\right) \pi^{y}(1-\pi)^{m-y}, \quad y=0,1, \ldots, m .
$$

Reparametrizando essa distribuição pelo seu valor esperado, tem-se que:

$$
E Y=\mu=m \pi \Rightarrow \pi=\frac{\mu}{m} .
$$

Substituindo, fica:

$$
p(y, \mu)=\left(\begin{array}{c}
m \\
y
\end{array}\right)\left(\frac{\mu}{m}\right)^{y}\left(1-\frac{\mu}{m}\right)^{m-y}=\left(\begin{array}{c}
m \\
y
\end{array}\right)\left(\frac{1}{m}\right)^{m} \mu^{y}(m-\mu)^{m-y},
$$

para $y=0,1, \ldots, m$.

O logaritmo da função de verossimilhança para o modelo sob pesquisa é

$$
l(y, \mu)=\ln \left(\begin{array}{c}
m \\
y
\end{array}\right)+\ln \left(\frac{1}{m}\right)^{m}+y \ln \mu+(m-y) \ln (m-\mu),
$$

e para o modelo superparametrizado é 


$$
l(y, y)=\ln \left(\begin{array}{c}
m \\
y
\end{array}\right)+\ln \left(\frac{1}{m}\right)^{m}+y \ln y+(m-y) \ln (m-y) .
$$

Daí, a deviance é calculada por:

$$
\begin{aligned}
2(l(y, y)-l(y, \mu)) & =2[y \ln y+(m-y) \ln (m-y)-y \ln \mu-(m-y) \ln (m-\mu)] \\
& =2\left\{y \ln \frac{y}{\mu}+(m-y) \ln \frac{(m-y)}{(m-\mu)}\right\} .
\end{aligned}
$$

Portanto, a deviance para uma v.a. $Y \sim b(m, p)$ é dada por

$$
D(y, \hat{\mu})=D(y, m, p)=2\left\{y \ln \frac{y}{\hat{\mu}}+(m-y) \ln \frac{(m-y)}{(m-\hat{\mu})}\right\}
$$

onde $\hat{\mu}$ é o valor médio estimado do modelo sob pesquisa.

A deviance tem uma distribuição desconhecida, mas no caso de grandes amostras, ela apresenta uma distribuição assintoticamente $\chi_{n-p}^{2}$, onde $n-p$ é o número de graus de liberdade do modelo, sendo $n=$ número de variáveis e $p=$ número de parâmetros envolvidos no modelo, mas essa aproximação apresenta um resto da ordem de $O\left(m^{3 / 2}\right)$, portanto ela não é boa, principalmente no caso de pequenas amostras.

\subsection{Expansão da Deviance sem correção}

\subsubsection{Para uma variável}

Como visto anteriormente, obtida a deviance, McCullagh e Nelder (1991) citam que sua distribuição é aproximadamente $\chi^{2}$ com erro da ordem de $O\left(m^{-3 / 2}\right)$. Se a verdadeira distribuição da deviance fosse $\chi^{2}$, os momentos calculados para a deviance deveriam coincidir com os momentos da distribuição $\chi^{2}$.

Assim, dada a variável aleatória $Y \sim b(m, p)$, tem-se que sua deviance é dada por (24), e seu valor esperado é dado por: 


$$
E(D(Y, \hat{\mu}))=2 \sum_{y=1}^{m-1}\left[y \ln \frac{y}{\hat{\mu}}+(m-y) \ln \frac{m-y}{m-\hat{\mu}}\right]\left(\begin{array}{c}
m \\
y
\end{array}\right) p^{y}(1-p)^{(m-y)}
$$

que não tem valor explícito. Ocorre nesta expressão que, $y$ toma valores de 1 até $m-1$ pois, quando $y=0 \mathrm{em} \ln (y / \hat{\mu})$ e quando $y=m \mathrm{em} \ln [(m-y) /(m-\hat{\mu})]$, o valor esperado não existe. A fim de obter uma forma aproximada para o valor esperado da deviance, seja

$$
D(y, m, p)=g(y)
$$

e expandindo essa função em série de Taylor em torno do ponto $\mu$, segue que $g(y)$ terá a forma

$$
\begin{aligned}
g(y) & =g(\mu)+g^{\prime}(\mu)(y-\mu)+g^{\prime \prime}(\mu) \frac{(y-\mu)^{2}}{2 !}+g^{\prime \prime \prime}(\mu) \frac{(y-\mu)^{3}}{3 !}+ \\
& +g^{i v}(\mu) \frac{(y-\mu)^{4}}{4 !}+\cdots
\end{aligned}
$$

Fazendo os cálculos, tem-se:

$$
\begin{gathered}
g(\mu)=2\left[\mu \ln \frac{\mu}{\mu}+(m-\mu) \ln \frac{m-\mu}{m-\mu}\right]=0 \\
g^{\prime}(y)=2\left[\ln \frac{y}{m p}-\ln \frac{m-y}{m-m p}\right] \Rightarrow g^{\prime}(\mu)=2\left[\ln \frac{m p}{m p}-\ln \frac{m-m p}{m-m p}\right]=0 \\
g^{\prime \prime}(y)=\frac{2}{y}+\frac{2}{m-y} \Rightarrow g^{\prime \prime}(\mu)=\frac{2}{m p}+\frac{2}{m-m p} \\
g^{\prime \prime \prime}(y)=-\frac{2}{y^{2}}+\frac{2}{(m-y)^{2}} \Rightarrow g^{\prime \prime \prime}(\mu)=-\frac{2}{m^{2} p^{2}}+\frac{2}{(m-m p)^{2}} \\
g^{i v}(y)=\frac{4}{y^{3}}+\frac{4}{(m-y)^{3}} \Rightarrow g^{i v}(\mu)=\frac{4}{m^{3} p^{3}}+\frac{4}{(m-m p)^{3}} .
\end{gathered}
$$


Portanto, substituindo as derivadas de $g(y)$ em (25) e calculando o valor esperado para uma variável aleatória $Y \sim b(m, p)$, vem

$$
\begin{gathered}
E(g(Y))=0+0+\left(\frac{2}{m p}+\frac{2}{m(1-p)}\right) \frac{m p(1-p)}{2 !}+ \\
+\left(\frac{-2}{m^{2} p^{2}}+\frac{2}{m^{2}(1-p)^{2}}\right)\left[\frac{m p-3 m p^{2}+2 m p^{3}}{3 !}\right]+ \\
+\left(\frac{4}{m^{3} p^{3}} \frac{4}{m^{3}(1-p)^{3}}\right)\left[\frac{m p-7 m p^{2}+12 m p^{3}-6 m p^{4}+3 m^{2} p^{2}(1-p)^{2}}{4 !}\right]+\cdots
\end{gathered}
$$

Simplificando, tem-se que

$$
E(g(Y))=E(D(Y, m, p))=1+\frac{1}{6 m}\left[\frac{1-p+p^{2}}{p(1-p)}\right]+O\left(m^{-2}\right)
$$

e pode-se observar que existe um viés no valor esperado da deviance da ordem de $m^{-1}$.

\subsubsection{Para $n$ variáveis}

Se considerarmos agora variáveis aleatórias $Y_{1}, \ldots, Y_{n}$ independentes e identicamente distribuídas com distribuição $b(m, p)$, a deviance envolvendo as $n$ variáveis é dada por:

$$
D(\mathbf{y}, m, p)=2 \sum_{i=1}^{n}\left(y_{i} \ln \frac{y_{i}}{\hat{\mu}}+\left(m-y_{i}\right) \ln \frac{m-y_{i}}{m-\hat{\mu}}\right)
$$

e o valor esperado da deviance é dado por:

$$
E(D(\mathbf{y}, m, p))=2 \sum_{i=1}^{n}\left(y_{i} \ln \frac{y_{i}}{\hat{\mu}}+\left(m-y_{i}\right) \ln \frac{m-y_{i}}{m-\hat{\mu}}\right)\left(\begin{array}{c}
m \\
y
\end{array}\right) p^{y_{i}}(1-p)^{\left(m-y_{i}\right)}
$$

Para este caso, o modelo sendo ajustado é o modelo de média e portanto $\hat{\mu}=\bar{y}$. Logo, 


$$
D(\mathbf{y}, m, p)=2 \sum_{i=1}^{n}\left(y_{i} \ln \frac{n y_{i}}{\sum_{i=1}^{n} y_{i}}+\left(m-y_{i}\right) \ln \frac{n\left(m-y_{i}\right)}{n m-\sum_{i=1}^{n} y_{i}}\right)
$$

A fim de obter uma forma aproximada para o valor esperado da deviance, considere

$$
D(\mathbf{y}, m, p)=g(\mathbf{y})
$$

e vê-se que $g(\boldsymbol{\mu})=0$.

Expandindo essa função em série de Taylor em torno do ponto $\mu$, segue que $g(\mathbf{y})$ terá a forma

$$
\begin{aligned}
g(\mathbf{y}) & =g(\boldsymbol{\mu})+d g(\boldsymbol{\mu})(\mathbf{y}-\boldsymbol{\mu})+\frac{1}{2 !} d^{2} g(\boldsymbol{\mu})(\mathbf{y}-\boldsymbol{\mu})^{2}+\frac{1}{3 !} d^{3} g(\boldsymbol{\mu})(\mathbf{y}-\boldsymbol{\mu})^{3}+\cdots \\
& =g(\mu)+\sum_{i=1}^{n} \frac{\partial g}{\partial y_{i}}\left(Y_{i}-\mu\right)+\frac{1}{2 !} \sum_{i=1}^{n} \frac{\partial^{2} g}{\partial y_{i}^{2}}\left(Y_{i}-\mu\right)^{2}+\frac{1}{3 !} \sum_{i=1}^{n} \frac{\partial^{3} g}{\partial y_{i}^{3}}\left(Y_{i}-\mu\right)^{3}+ \\
& +\frac{1}{4 !} \sum_{i=1}^{n} \frac{\partial^{4} g}{\partial y_{i}^{4}}\left(Y_{i}-\mu\right)^{4}+\frac{1}{2 ! 2 !} \sum_{i<j=2}^{n} \frac{\partial^{4} g}{\partial y_{i}^{2} \partial y_{j}^{2}}\left[\left(Y_{i}-\mu\right)^{2}\left(Y_{j}-\mu\right)^{2}\right]+\cdots
\end{aligned}
$$

Obtendo as derivadas de $1^{\underline{a}}, 2^{\underline{a}}, 3^{\underline{a}}$ e $4^{\underline{\underline{a}}}$ ordem de $g$ em relação a $y_{1}$ tem-se:

$$
\begin{gathered}
\frac{\partial g}{\partial y_{1}}=2\left[\ln \frac{y_{1}}{\bar{y}}-\frac{y_{1}}{n \bar{y}}-\ln \frac{m-y_{1}}{m-\bar{y}}+\frac{m-y_{1}}{n(m-\bar{y})}\right] \\
\frac{\partial^{2} g}{\partial y_{1}^{2}}=2\left[\frac{1}{y_{1}}-\frac{1}{n \bar{y}}+\frac{1}{m-y_{1}}-\frac{1}{n(m-\bar{y})}\right] \\
\frac{\partial^{3} g}{\partial y_{1}^{3}}=2\left[-\frac{1}{y_{1}^{2}}+\frac{1}{n(\bar{y})^{2}}+\frac{1}{\left(m-y_{1}\right)^{2}}-\frac{1}{n(m-\bar{y})^{2}}\right] \\
\frac{\partial^{4} g}{\partial y_{1}^{4}}=2\left[\frac{2}{y_{1}^{3}}-\frac{2}{n(\bar{y})^{3}}+\frac{2}{\left(m-y_{1}\right)^{3}}-\frac{2}{n(m-\bar{y})^{3}}\right] .
\end{gathered}
$$


Pode-se observar que as derivadas parciais mistas de $2^{\underline{a}}$ ordem e $3^{\underline{a}}$ ordem envolvem termos da forma $\left(y_{1}-\mu\right)\left(y_{2}-\mu\right),\left(y_{1}-\mu\right)\left(y_{2}-\mu\right)^{2}$ que possuem valor esperado nulo. Mas isto não ocorre com a derivada de $4 \underline{\underline{a}}$ ordem que envolve fatores da forma $\left(y_{1}-\mu\right)^{2}\left(y_{2}-\mu\right)^{2}$, cujo valor esperado é o quadrado da variância. Desse modo a derivada de $4^{\underline{a}}$ ordem mista de $g$ para $y_{1}$ e $y_{2}$ é:

$$
\frac{\partial^{4} g}{\partial y_{1}^{2} \partial y_{2}^{2}}=\frac{4}{n^{3}}\left[-\frac{1}{(\bar{y})^{3}}-\frac{1}{(m-\bar{y})^{3}}\right] \text {. }
$$

Considerando agora as $n$ variáveis, tem-se:

$$
\begin{gathered}
\sum_{i=1}^{n} \frac{\partial g}{\partial y_{i}}=2\left[\sum_{i=1}^{n} \ln \frac{y_{i}}{\bar{y}}-\sum_{i=1}^{n} \ln \frac{m-y_{i}}{m-\bar{y}}\right] \\
\sum_{i=1}^{n} \frac{\partial^{2} g}{\partial y_{i}^{2}}=2\left[\sum_{i=1}^{n} \frac{1}{y_{i}}-\frac{1}{\bar{y}}+\sum_{i=1}^{n} \frac{1}{m-y_{i}}-\frac{1}{m-\bar{y}}\right] \\
\sum_{i=1}^{n} \frac{\partial^{3} g}{\partial y_{i}^{3}}=2\left[-\sum_{i=1}^{n} \frac{1}{\left(y_{i}\right)^{2}}+\frac{1}{(\bar{y})^{2}}+\sum_{i=1}^{n} \frac{1}{\left(m-y_{i}\right)^{2}}-\frac{1}{(m-\bar{y})^{2}}\right] \\
\sum_{i=1}^{n} \frac{\partial^{4} g}{\partial y_{i}^{4}}=4\left[\sum_{i=1}^{n} \frac{1}{\left(y_{i}\right)^{3}}-\frac{1}{(\bar{y})^{3}}+\sum_{i=1}^{n} \frac{1}{\left(m-y_{i}\right)^{3}}-\frac{1}{(m-\bar{y})^{3}}\right] .
\end{gathered}
$$

No caso da derivada mista, tem-se que como a soma de $n$ derivadas mistas é $\frac{n(n-1)}{2}$, fica:

$$
\sum_{i<j=2}^{n} \frac{\partial^{4} g}{\partial y_{i}^{2} \partial y_{j}^{2}}=2 \frac{n-1}{n^{2}}\left[-\frac{1}{(\bar{y})^{3}}-\frac{1}{(m-\bar{y})^{3}}\right] .
$$

Fazendo $y_{i}$ e $y_{j}$ iguais a $m p$, tem-se:

$$
\begin{gathered}
\left.\sum_{i=1}^{n} \frac{\partial g}{\partial y_{i}}\right|_{y_{i}=m p}=2\left[\sum_{i=1}^{n} \ln \frac{m p}{m p}-\sum_{i=1}^{n} \frac{m-m p}{m-m p}\right]=0 \\
\left.\sum_{i=1}^{n} \frac{\partial^{2} g}{\partial y_{i}^{2}}\right|_{y_{i}=m p}=2(n-1)\left[\frac{1}{m p}+\frac{1}{m-m p}\right]
\end{gathered}
$$




$$
\begin{gathered}
\left.\sum_{i=1}^{n} \frac{\partial^{3} g}{\partial y_{i}^{3}}\right|_{y_{i}=m p}=2(n-1)\left[-\frac{1}{(m p)^{2}}+\frac{1}{(m-m p)^{2}}\right] \\
\left.\sum_{i=1}^{n} \frac{\partial^{4} g}{\partial y_{i}^{4}}\right|_{y_{i}=m p}=4(n-1)\left[\frac{1}{(m p)^{3}}+\frac{1}{(m-m p)^{3}}\right] \\
\left.\sum_{i<j=2}^{n} \frac{\partial^{4} g}{\partial y_{i}^{2} \partial y_{j}^{2}}\right|_{y_{i}, y_{j}=m p}=\frac{2(n-1)}{n^{2}}\left[-\frac{1}{(m p)^{3}}-\frac{1}{(m-m p)^{3}}\right] .
\end{gathered}
$$

Substituindo as derivadas de $g(\mathbf{y})$ em (26) e calculando seu valor esperado para $n$ variáveis aleatórias $Y_{i} \sim b(m, p)$, vem:

$$
\begin{gathered}
E(g(\mathbf{Y}))=0+0+2(n-1)\left[\frac{1}{m p}+\frac{1}{m-m p}\right] \frac{m p(1-p)}{2 !}+ \\
+2(n-1)\left[-\frac{1}{(m p)^{2}}+\frac{1}{(m-m p)^{2}}\right] \frac{m p(1-p)(1-2 p)}{3 !}+ \\
+4(n-1)\left[\frac{1}{(m p)^{3}}+\frac{1}{(m-m p)^{3}}\right] \frac{m p(1-p)(1-6 p(1-p))+3 m^{2} p^{2}(1-p)^{2}}{4 !}+ \\
+\frac{2(n-1)}{n^{2}}\left[-\frac{1}{(m p)^{3}}-\frac{1}{(m-m p)^{3}}\right] \frac{m^{2} p^{2}(1-p)^{2}}{2 ! 2 !}+\cdots= \\
=(n-1)+\frac{1}{6} \frac{\left(n^{2}-n^{2} p+n^{2} p^{2}-3+9 p-9 p^{2}\right)(n-1)}{m p(1-p) n^{2}}+O\left(m^{-2}\right)
\end{gathered}
$$

onde novamente observa-se um viés no valor esperado da deviance da ordem de $m^{-1}$. 


\subsection{Expansão da Deviance considerando o Fator de Correção}

\subsubsection{Para uma Variável}

Se a distribuição da deviance é aproximadamente qui-quadrado com 1 grau de liberdade, espera-se que seu valor esperado seja aproximadamente 1. Mas, observa-se que existe um viés da ordem de $m^{-1}$, que tende a zero somente quando $m$ torna-se grande. Assim, para que se tenha uma melhor aproximação do valor esperado de 1 , é necessário que os termos de $O\left(m^{-1}\right)$ se anulem. Para isto, propõe-se uma correção para a variável resposta de modo que se reduza a ordem de convergência do valor esperado. Isso garantiria uma melhor aproximação da distribuição da deviance a uma distribuição qui-quadrado.

Desse modo, substituindo

$$
\begin{gathered}
y \rightarrow y+a \\
m \rightarrow m+2 a
\end{gathered}
$$

ainda com a suposição de que $Y+a \sim b(m, p)$, é preciso obter o valor de $a$ de modo a zerar o termo de $O\left(m^{-1}\right)$.

A deviance para a variável corrigida $Y+a$ será dada por

$$
D(y+a, m+2 a, p)=2\left[(y+a) \ln \frac{y+a}{m p}+(m+a-y) \ln \frac{m+a-y}{m+2 a-m p}\right]
$$

e considere novamente:

$$
D(y+a, m+2 a, p)=g(y) .
$$

Expandindo $g(y)$ em série de Taylor em torno do ponto $\mu=m p$, tem-se que

$$
g(\mu)=0
$$




$$
\begin{gathered}
g^{\prime}(y)=2\left[\ln \frac{y+a}{m p}-\ln \frac{m+a-y}{m+2 a-m p}\right] \Rightarrow g^{\prime}(\mu)=0 \\
g^{\prime \prime}(y)=\frac{2}{y+a}+\frac{2}{m+a-y} \Rightarrow g^{\prime \prime}(\mu)=\frac{2}{(m p)}+\frac{2}{m+2 a-m p} \\
g^{\prime \prime \prime}(y)=-\frac{2}{(y+a)^{2}}+\frac{2}{(m+a-y)^{2}} \Rightarrow g^{\prime \prime \prime}(\mu)=-\frac{2}{(m p)^{2}}+\frac{2}{(m+2 a-m p)^{2}} \\
g^{i v}(y)=\frac{4}{(y+a)^{3}}+\frac{4}{(m+a-y)^{3}} \Rightarrow g^{i v}(\mu)=\frac{4}{(m p)^{3}}+\frac{4}{(m+2 a-m p)^{3}} .
\end{gathered}
$$

Aplicando o valor esperado na expansão, considerando agora a variável aleatória $Y$, tem-se que

$$
\begin{aligned}
& E(D(Y+a, m+2 a, p))=0+0 . E(Y-\mu)+\left[\frac{2}{(m p)}+\frac{2}{m+2 a-m p}\right] \frac{E(Y-\mu)^{2}}{2 !}+ \\
& +\left[-\frac{2}{(m p)^{2}}+\frac{2}{(m+2 a-m p)^{2}}\right] \frac{E(Y-\mu)^{3}}{3 !}+\left[\frac{4}{(m p)^{3}}+\frac{4}{(m+2 a-m p)^{3}}\right] \frac{E(Y-\mu)^{4}}{4 !}+\cdots \\
& =\left[\frac{2}{m p}+\frac{2}{m+2 a-m p}\right] \frac{m p(1-p)}{2 !}+\left[-\frac{2}{m^{2} p^{2}}+\frac{2}{(m+2 a-m p)^{2}}\right] \frac{m p(1-p)(1-2 p)}{3 !}+ \\
& +\left[\frac{4}{m^{3} p^{3}}+\frac{4}{(m+2 a-m p)^{3}}\right] \frac{3 m^{2} p^{2}(1-p)^{2}+m p(1-p)(1-6 p(1-p))}{4 !}+\cdots= \\
& =1-p-\frac{1-3 p+2 p^{2}}{3 m p}+\frac{(1-p)^{2}}{2 m p}+\frac{m p(1-p)}{m+2 a-m p}+\frac{m p\left(1-3 p+2 p^{2}\right)}{3(m+2 a-m p)^{2}}+ \\
& +\frac{m^{2} p^{2}(1-p)^{2}}{2(m+2 a-m p)^{3}}+O\left(m^{-2}\right) .
\end{aligned}
$$

Expandindo cada termo da expansão acima em série de Taylor, em torno do ponto $a$, segue que: 


$$
\begin{aligned}
E(D(Y+a, m+2 a, p)) & =1-\frac{2 a p}{m-m p}+\frac{m p\left(1-3 p+2 p^{2}\right)}{3(m-m p)^{2}}+\frac{m^{2} p^{2}(1-p)^{2}}{2(m-m p)^{3}}+ \\
& +-\frac{1-3 p+2 p^{2}}{3 m p}+\frac{(1-p)^{2}}{2 m p}+O\left(m^{-2}\right) \\
& =1-\frac{1}{6} \frac{12 a p^{2}-1+p-p^{2}}{m p(1-p)}+O\left(m^{-2}\right) .
\end{aligned}
$$

A fim de reduzir a ordem de convergência na expansão da deviance, calcula-se o valor de $a$ igualando o termo da ordem de $O\left(m^{-1}\right)$ a zero, isto é

$$
\begin{gathered}
-\frac{1}{6} \frac{12 a p^{2}-1+p-p^{2}}{m p(1-p)}=0 \\
12 a p^{2}=1-p+p^{2} \\
a=\frac{1-p+p^{2}}{12 p^{2}} .
\end{gathered}
$$

obtendo assim o valor de $a$ que reduz a ordem de convergência na expansão do valor esperado da deviance, para variáveis com distribuição binomial de parâmetros $m$ e $p$.

\subsubsection{Para $n$ Variáveis}

Neste caso, se a distribuição da deviance é assintoticamente $\chi^{2}$, o seu valor esperado deveria ser aproximadamente $(n-1)$. Novamente, observa-se que existe um viés da ordem de $m^{-1}$, que tende a zero somente quando $m$ torna-se grande. Assim, para que se tenha uma melhor aproximação do valor esperado de $(n-1)$, é necessário que os termos de $O\left(m^{-1}\right)$ se anulem.

Novamente a fim de reduzir a ordem de convergência da deviance e torná-la mais próxima a uma distribuição de qui-quadrado, introduz-se uma correção aos valores observados, denotada por $a$ e, do mesmo modo, substitui-se $y$ por $y+a \mathrm{e}$ $m$ por $m+2 a$. Assim, a fórmula da deviance com a correção ficará da seguinte forma: 


$$
D(\mathbf{y}+a, m+2 a, p)=2 \sum_{i=1}^{n}\left[\left(y_{i}+a\right) \ln \frac{y_{i}+a}{\bar{y}+a}+\left(m+a-y_{i}\right) \ln \frac{m+a-y_{i}}{m+a-\bar{y}}\right]
$$

e considere $D(\mathbf{y}+a, m+2 a, p)=g(\mathbf{y})$. Expandindo $g(\mathbf{y})$ em série de Taylor em torno do ponto $\mu=\bar{y}$, calculam-se as derivadas de $g$ em relação a $y_{1}$, que são:

$$
\begin{gathered}
\frac{\partial g}{\partial y_{1}}=2\left[\ln \frac{y_{1}+a}{\bar{y}+a}-\frac{y_{1}+a}{n(\bar{y}+a)}-\ln \frac{m+a-y_{1}}{m+a-\bar{y}}+\frac{m+a-y_{1}}{n(m+a-\bar{y})}\right] \\
\frac{\partial^{2} g}{\partial y_{1}^{2}}=2\left[\frac{1}{y_{1}+a}-\frac{1}{n(\bar{y}+a)}+\frac{1}{m+a-y_{1}}-\frac{1}{n(m+a-\bar{y})}\right] \\
\frac{\partial^{3} g}{\partial y_{1}^{3}}=2\left[-\frac{1}{\left(y_{1}+a\right)^{2}}+\frac{1}{n(\bar{y}+a)^{2}}+\frac{1}{\left(m+a-y_{1}\right)^{2}}-\frac{1}{n(m+a-\bar{y})^{2}}\right] \\
\frac{\partial^{4} g}{\partial y_{1}^{4}}=2\left[\frac{2}{\left(y_{1}+a\right)^{3}}-\frac{2}{n(\bar{y}+a)^{3}}+\frac{2}{\left(m+a-y_{i}\right)^{3}}-\frac{2}{n(m+a-\bar{y})^{3}}\right] \\
\frac{\partial^{4} g}{\partial y_{1}^{2} \partial y_{2}^{2}}=\frac{4}{n^{3}}\left[-\frac{1}{(\bar{y}+a)^{3}}-\frac{1}{(m+a-\bar{y})^{3}}\right] \cdot
\end{gathered}
$$

Considerando agora as $n$ variáveis, tem-se:

$$
\begin{gathered}
\sum_{i=1}^{n} \frac{\partial g}{\partial y_{i}}=2\left[\sum_{i=1}^{n} \ln \frac{y_{i}+a}{\bar{y}+a}-\sum_{i=1}^{n} \ln \frac{m+a-y_{i}}{m+a-\bar{y}}\right] \\
\sum_{i=1}^{n} \frac{\partial^{2} g}{\partial y_{i}^{2}}=2\left[\sum_{i=1}^{n} \frac{1}{y_{i}+a}-\frac{1}{\bar{y}+a}+\sum_{i=1}^{n} \frac{1}{m+a-y_{i}}-\frac{1}{m+a-\bar{y}}\right] \\
\sum_{i=1}^{n} \frac{\partial^{3} g}{\partial y_{i}^{3}}=2\left[-\sum_{i=1}^{n} \frac{1}{\left(y_{i}+a\right)^{2}}+\frac{1}{(\bar{y}+a)^{2}}+\sum_{i=1}^{n} \frac{1}{\left(m+a-y_{i}\right)^{2}}-\frac{1}{(m+a-\bar{y})^{2}}\right] \\
\sum_{i=1}^{n} \frac{\partial^{4} g}{\partial y_{i}^{4}}=4\left[\sum_{i=1}^{n} \frac{1}{\left(y_{i}+a\right)^{3}}-\frac{1}{(\bar{y}+a)^{3}}+\sum_{i=1}^{n} \frac{1}{\left(m+a-y_{i}\right)^{3}}-\frac{1}{(m+a-\bar{y})^{3}}\right] .
\end{gathered}
$$


Neste caso, a soma de $n$ derivadas mistas é $\frac{n(n-1)}{2}$, então:

$$
\sum_{i<j=2}^{n} \frac{\partial^{4} g}{\partial y_{i}^{2} \partial y_{j}^{2}}=\frac{2(n-1)}{n^{2}}\left[-\frac{1}{(\bar{y}+a)^{3}}-\frac{1}{(m+a-\bar{y})^{3}}\right] .
$$

Daí, as derivadas em relação a $y_{i}$ e $y_{j}$ no ponto $m p-a$ ficarão:

$$
\begin{gathered}
g(\mu)=0 \\
\left.\frac{\partial g}{\partial y_{i}}\right|_{y_{i}+a=m p}=2\left[\sum_{i=1}^{n} \ln \frac{m p}{m p}-\sum_{i=1}^{n} \ln \frac{m+2 a-m p}{m+2 a-m p}\right]=0 \\
\left.\frac{\partial^{2} g}{\partial y_{i}^{2}}\right|_{y_{i}+a=m p}=2(n-1)\left[\frac{1}{m p}+\frac{1}{m+2 a-m p}\right] \\
\left.\frac{\partial^{3} g}{\partial y_{i}^{3}}\right|_{y_{i}+a=m p}=2(n-1)\left[-\frac{1}{(m p)^{2}}+\frac{1}{(m+2 a-m p)^{2}}\right] \\
\left.\frac{\partial^{4} g}{\partial y_{i}^{4}}\right|_{y_{i}+a=m p}=4(n-1)\left[\frac{1}{(m p)^{3}}+\frac{1}{(m+2 a-m p)^{3}}\right] \\
\left.\frac{\partial^{4} g}{\partial y_{i}^{2} \partial y_{j}^{2}}\right|_{y_{i}+a, y_{j}+a=m p}=\frac{2(n-1)}{n^{2}}\left[-\frac{1}{(m p)^{3}}-\frac{1}{(m+2 a-m p)^{3}}\right] .
\end{gathered}
$$

Então, substituindo as derivadas na expansão de $g(\mathbf{y})$ e calculando seu valor esperado, tem-se:

$$
\begin{gathered}
E(g(\mathrm{Y}))=0+0+2(n-1)\left[\frac{1}{m p}+\frac{1}{m+2 a-m p}\right] \frac{m p(1-p)}{2 !}+ \\
+2(n-1)\left[-\frac{1}{(m p)^{2}}+\frac{1}{(m+2 a-m p)^{2}}\right] \frac{m p(1-p)(1-2 p)}{3 !}+ \\
+4(n-1)\left[\frac{1}{(m p)^{3}}+\frac{1}{(m+2 a-m p)^{3}}\right] \frac{\left[m p(1-p)(1-6 p(1-p))+3 m^{2} p^{2}(1-p)^{2}\right]}{4 !}+
\end{gathered}
$$




$$
+\frac{2(n-1)}{n^{2}}\left[-\frac{1}{(m p)^{3}}-\frac{1}{(m+2 a-m p)^{3}}\right] \frac{m^{2} p^{2}(1-p)^{2}}{2 ! 2 !}+\cdots
$$

Fazendo as simplificações necessárias e expandindo novamente em série de Taylor, agora para $a$, tem-se:

$$
\begin{gathered}
E(D(\mathbf{Y}+a, m+2 a, p))=(n-1)-\frac{2(n-1) p}{m(1-p)} a-\frac{1}{3} \frac{(1-2 p)^{2}(n-1)}{m p(1-p)}+ \\
+\frac{1}{2} \frac{(n-1)\left(1-3 p+3 p^{2}\right)}{m p(1-p)}-\frac{1}{2} \frac{\left(1-3 p+3 p^{2}\right)(n-1)}{n^{2} m p(1-p)}+\cdots \\
=(n-1)-\frac{1}{6} \frac{(n-1)\left(n^{2}\left(12 p^{2} a-1+p-p^{2}\right)-3\left(1-3 p+3 p^{2}\right)\right)}{n^{2} m p(1-p)}+O\left(m^{-2}\right) .
\end{gathered}
$$

Para reduzir a ordem de convergência na expansão da deviance, será obtido o valor de $a$, igualando o termo da ordem de $O\left(\mathrm{~m}^{-1}\right)$ a zero:

$$
\begin{aligned}
& 0=-\frac{1}{6} \frac{(n-1)\left(n^{2}\left(12 p^{2} a-1+p-p^{2}\right)-3\left(1-3 p+3 p^{2}\right)\right)}{n^{2} m p(1-p)} \\
& a=\frac{1-p+p^{2}}{12 p^{2}}-\frac{1-3 p+3 p^{2}}{4 n^{2} p^{2}}
\end{aligned}
$$

obtendo o valor de $a$ que reduz o viés da distribuição da deviance, aproximando-a da distribuição de qui-quadrado, cuja esperança é $(n-1)$ (número de graus de liberdade para $n$ variáveis).

Pode-se notar que quando $n \rightarrow+\infty$, o segundo termo da correção tende a zero. Assim, o valor da correção para $n$ variáveis se equipara ao valor da correção para uma variável quando $n$ é grande. 


\section{Resultados E Discussão}

Como visto anteriormente, tem-se que o valor esperado da deviance é dado por

$$
E(D(Y, \hat{\mu}))=2 \sum_{y=1}^{m-1}\left[Y \ln \frac{Y}{\hat{\mu}}+(m-Y) \ln \frac{m-Y}{m-\hat{\mu}}\right]\left(\begin{array}{c}
m \\
Y
\end{array}\right) p^{Y}(1-p)^{(m-Y)},
$$

quando $Y \sim b(m, p)$, que não possui valor explícito.

Considerando vários valores de $m$ e $p$, obteve-se esse valor esperado numericamente, cujos comportamentos são mostrados nas Figuras 1, 2, 3 e 4.

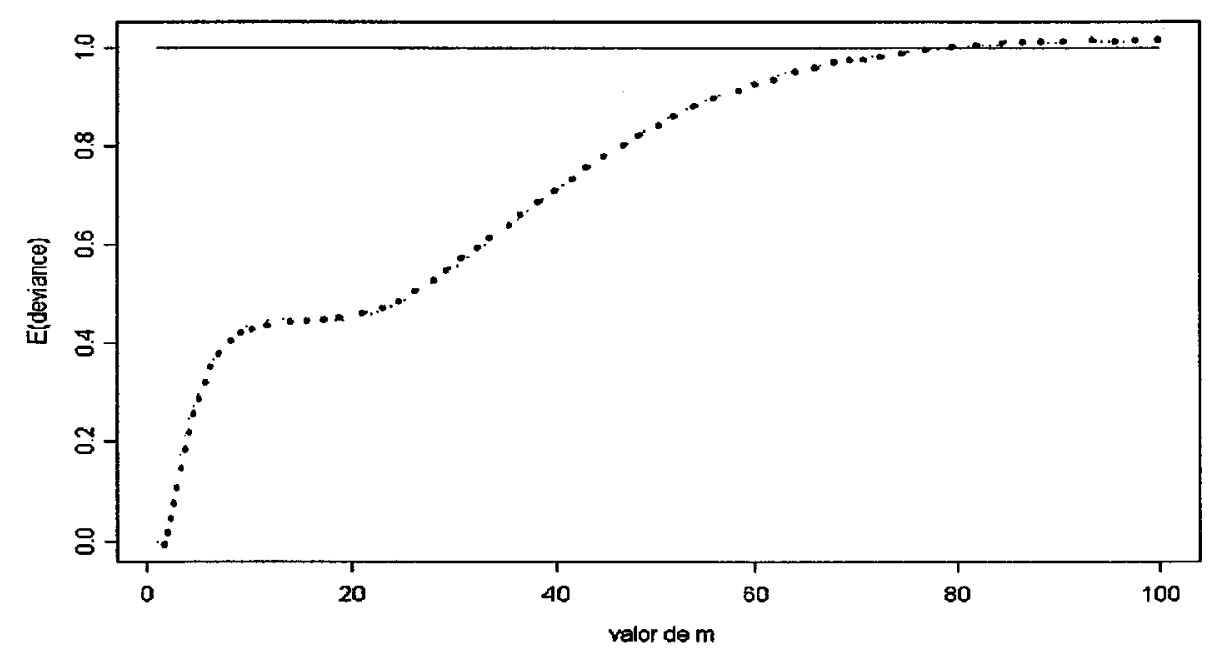

Figura 1: Gráfico da deviance da distribuição binomial para o modelo de média para $m=0(1) 100$ e $p=0,1$. 


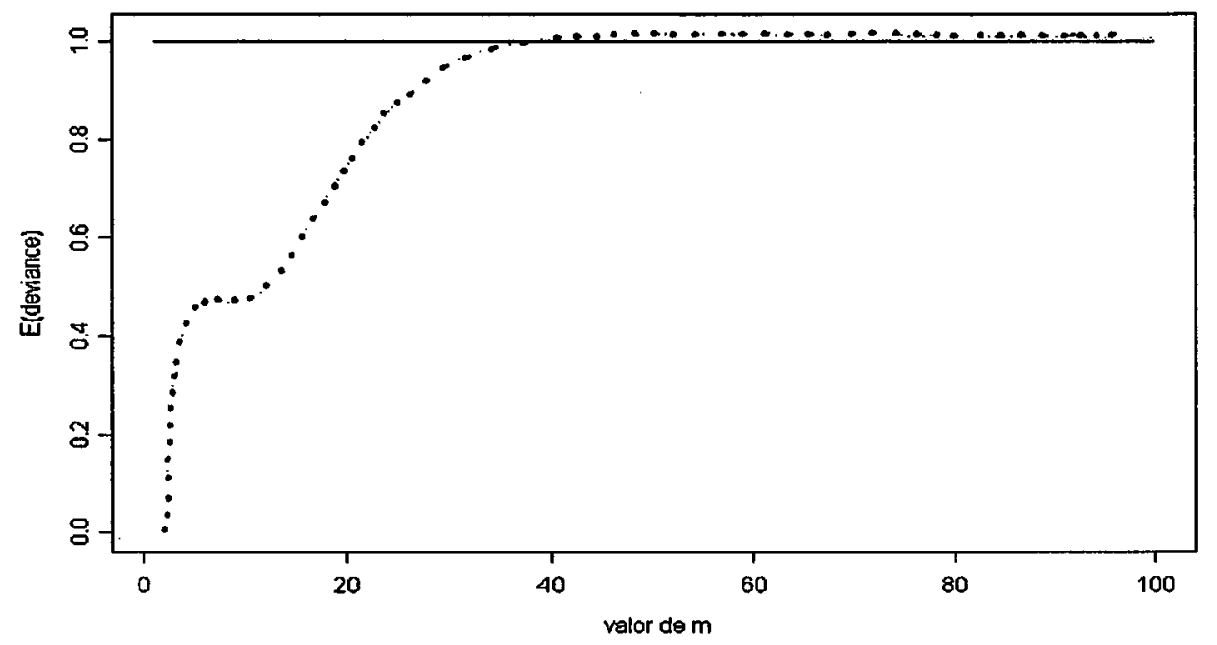

Figura 2: Gráfico da deviance da distribuição binomial para o modelo de média para $m=0(1) 100$ e $p=0,2$.

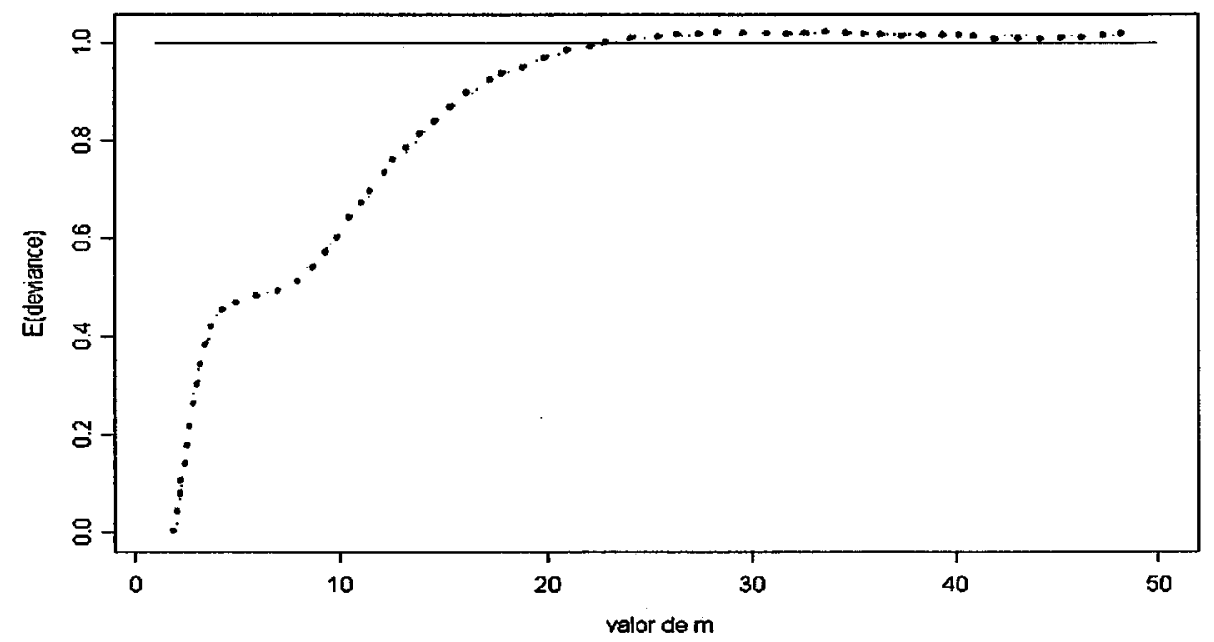

Figura 3: Gráfico da deviance da distribuição binomial para o modelo de média para $m=0(1) 50$ e $p=0,3$. 


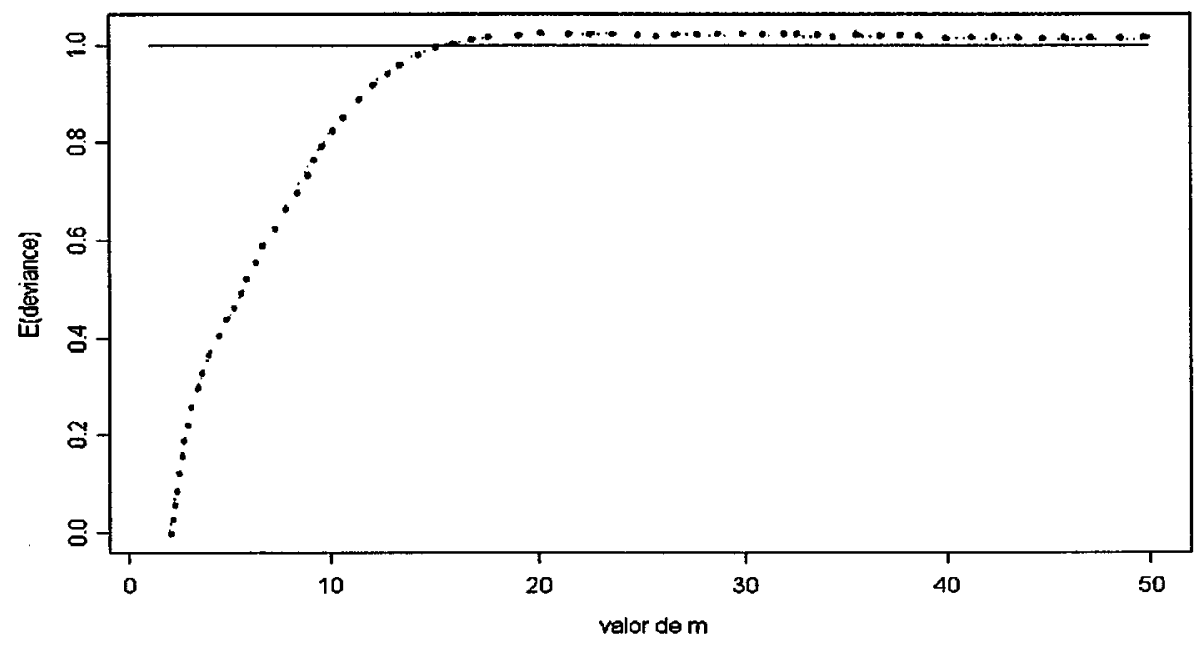

Figura 4: Gráfico da deviance da distribuição binomial para o modelo de média para $m=0(1) 50$ e $p=0,4$.

Nas Figuras 1 e 2, nota-se que quando o valor de $m$ é pequeno, a deviance não está próxima de 1 , o que pode indicar um mal ajuste do modelo sob pesquisa pela falta de ajuste da distribuição da deviance a uma distribuição $\chi^{2}$, isso também pelo fato de $p$ ser pequeno. Já, quando o valor de $p$ aumenta, o valor esperado da deviance já se aproxima mais de 1, como mostram as Figuras 3, 4 e 5.

Fazendo os mesmos cálculos para o valor esperado da deviance, considerando o fator de correção $a$ obtido anteriormente para diversos valores de $m$ e $p$, os comportamentos podem ser vistos pelas Figuras de 5 a 9 . 


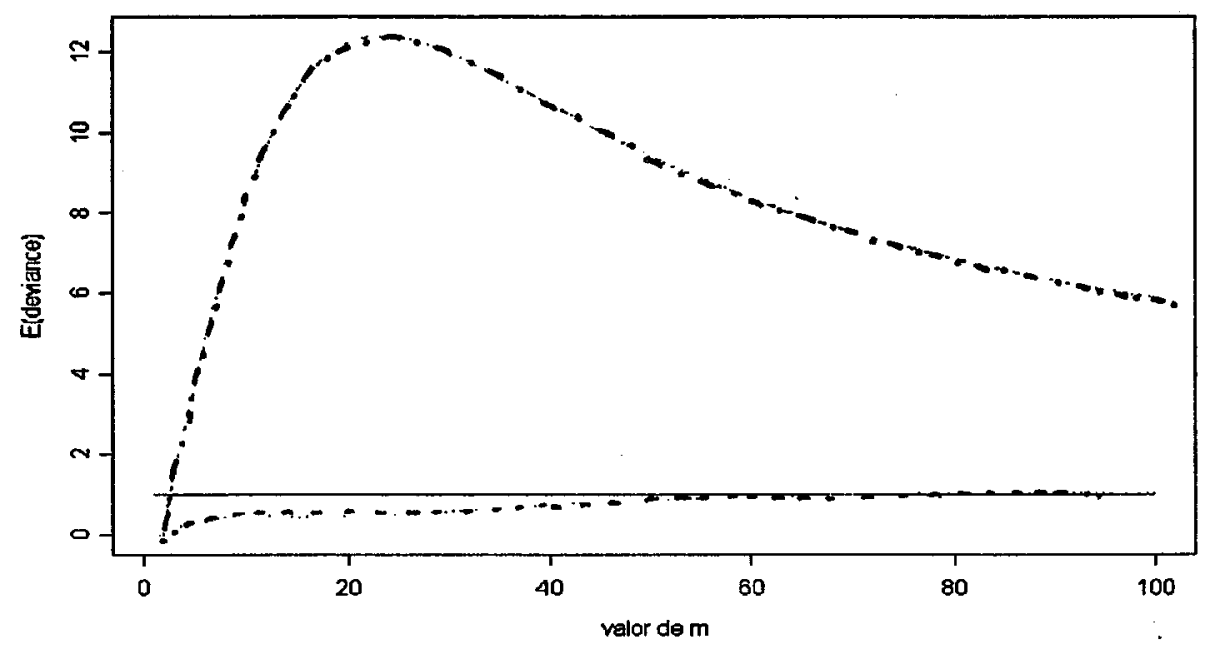

Figura 5: Gráfico da deviance da distribuição binomial para o modelo de média com e sem o fator de correção para $m=0(1) 100$ e $p=0,1$. ( - - . - . - - = deviance sem correção, - - - - - = deviance com correção)

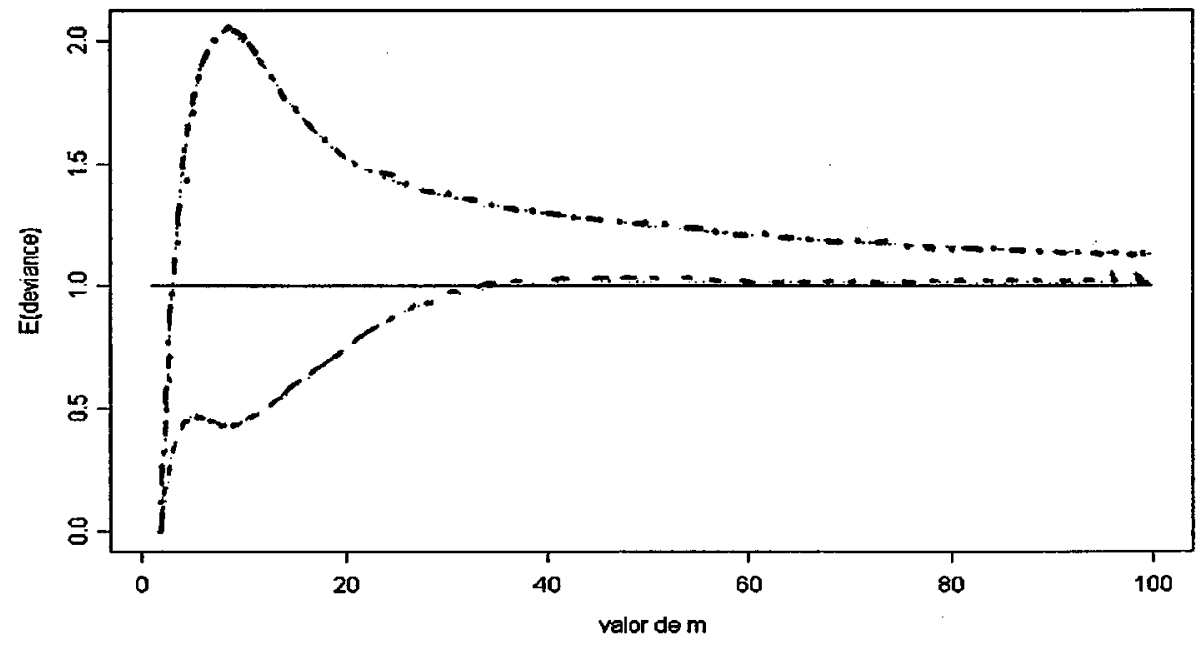

Figura 6: Gráfico da deviance da distribuição binomial para o modelo de média com e sem o fator de correção para $m=0(1) 100$ e $p=0,2 .(-\ldots+\cdots$ - . deviance sem correção, - - - - - = deviance com correção) 


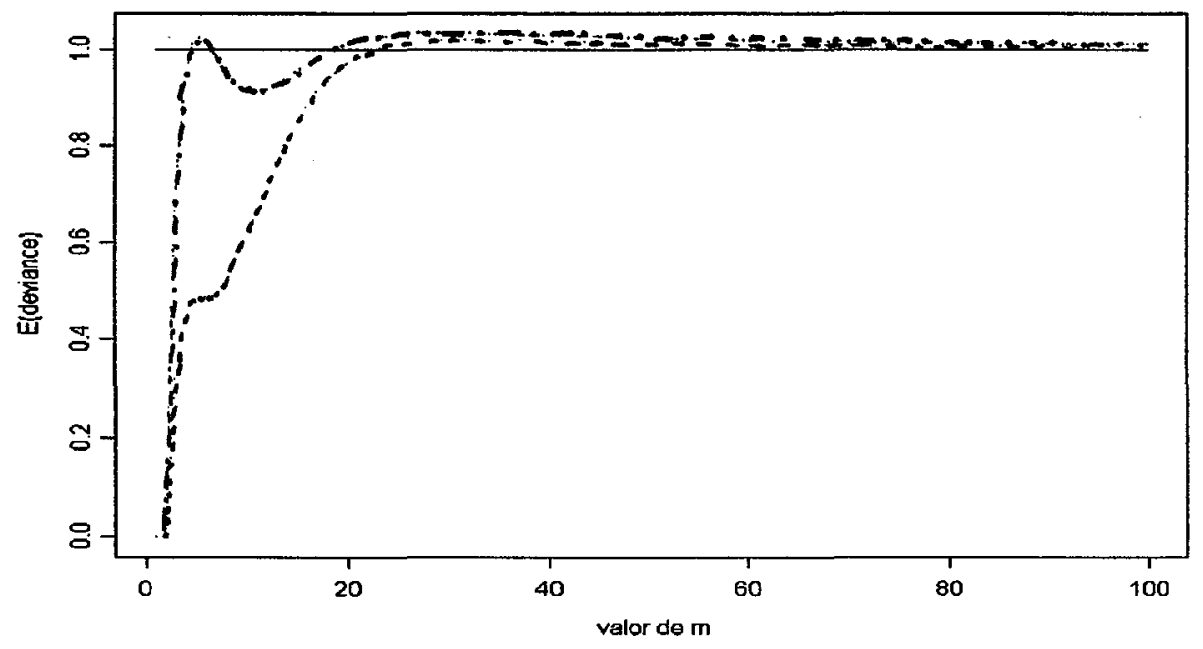

Figura 7: Gráfico da deviance da distribuição binomial para o modelo de média com e sem o fator de correção para $m=0(1) 100$ e $p=0,3 .(-\cdots-\cdots$ - - = deviance sem correção, - - - - - = deviance com correção)

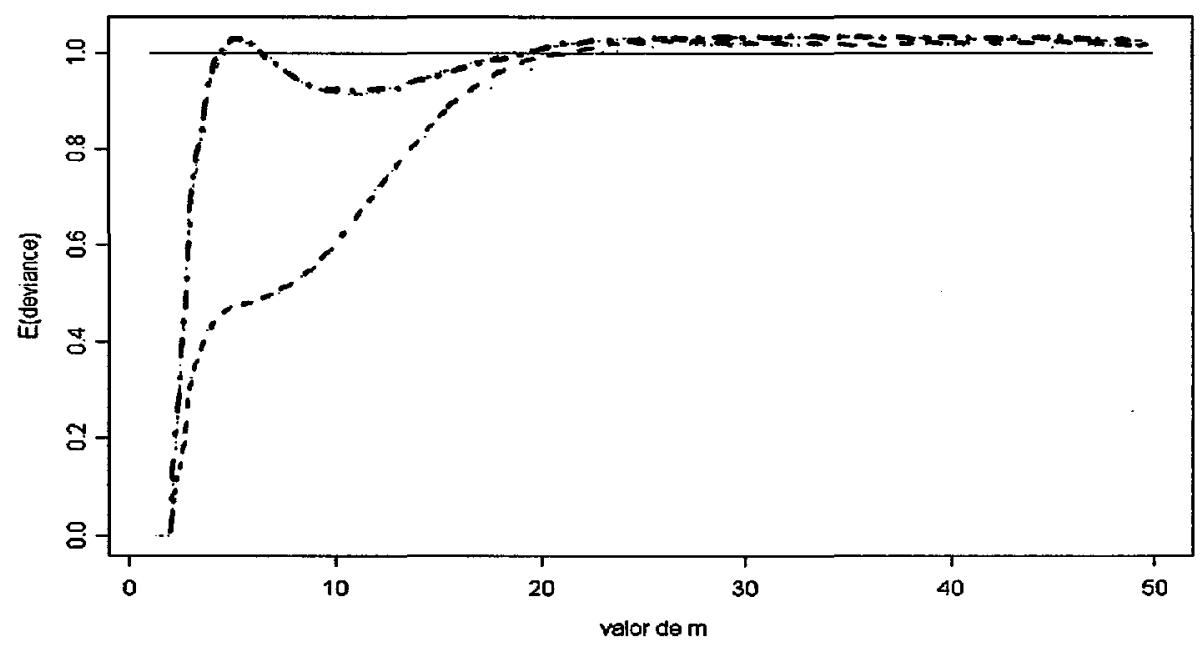

Figura 8: Gráfico da deviance da distribuição binomial para o modelo de média com e sem o fator de correção para $m=0(1) 50$ e $p=0,3 .(-\cdots-\cdots-\cdots$ deviance sem correção, - - - - - = deviance com correção) 


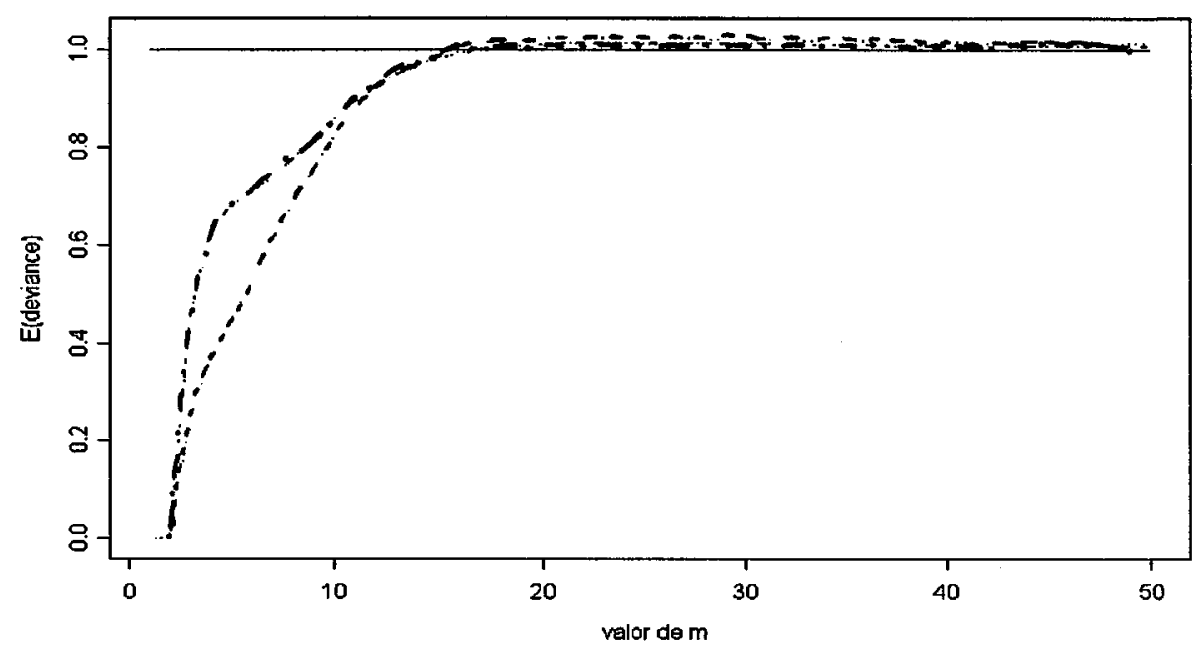

Figura 9: Gráfico da deviance da distribuição binomial para o modelo de média com e sem o fator de correção para $m=0(1) 50$ e $p=0,4 .(-\ldots \ldots$ - . - = deviance sem correção, - - - - - = deviance com correção)

Analisando as Figuras 5 e 6, onde $p=0,1$ e 0,2, tem-se que o valor de $a$ superestima o valor esperado da deviance. Como mostram as Figuras 7 e 8, quando $p=0,3$, o valor esperado teve sua melhor aproximação. Na Figura 9 , onde $p=0,4$, o valor de $a$ não afeta muito o valor esperado da deviance, indicando que a correção nesse caso, pode ser desnecessária. Como o interessante é obter um valor de $a$ que não dependa de $p$, tem-se que para $p=0,3$, o valor $a$ é 0,73 .

Assim, fazendo $a=0,73$, calcula-se novamente o valor esperado da deviance para vários valores de $m$ e $p$ e os comportamentos desses valores são mostrados nas Figuras 10 a 13. 


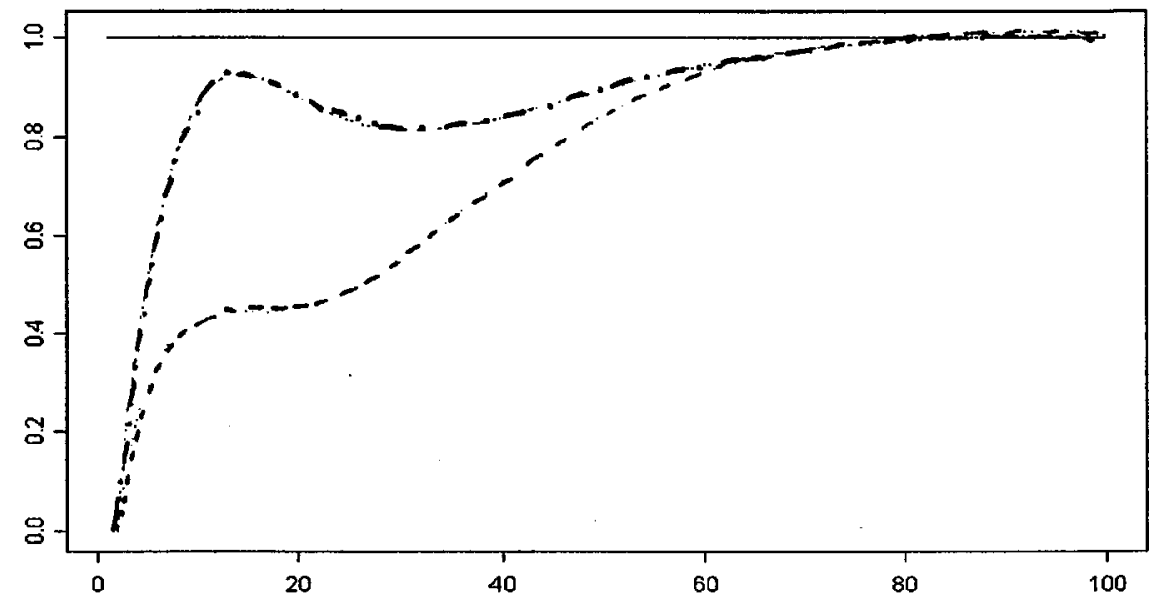

Figura 10: Gráfico da deviance da distribuição binomial para o modelo de média com e sem o fator de correção $a=0,73$ para $m=0(1) 100$ e $p=0,1$. (- - . - . - = deviance sem correção, - - - - = deviance com correção)

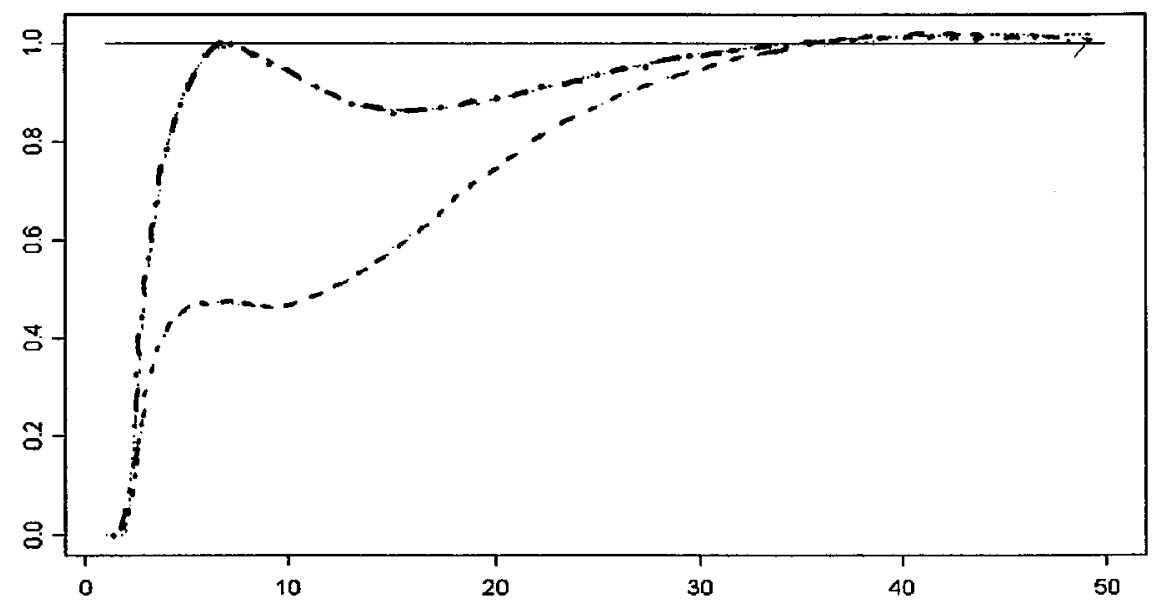

Figura 11: Gráfico da deviance da distribuição binomial para o modelo de média com e sem o fator de correção $a=0,73$ para $m=0(1) 50$ e $p=0,2$. (- . . - . - = deviance sem correção, - - - - = deviance com correção) 


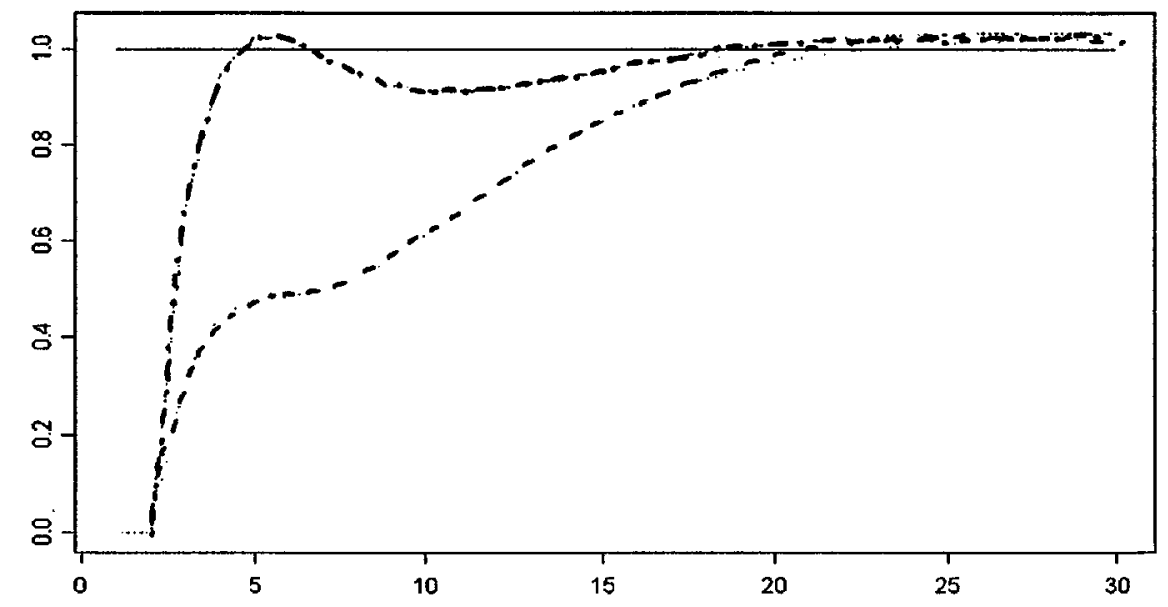

Figura 12: Gráfico da deviance da distribuição binomial para o modelo de média com e sem o fator de correção $a=0,73$ para $m=0(1) 30$ e $p=0,3$.

(- - - - . - . = deviance sem correção, - - - - - = deviance com correção)

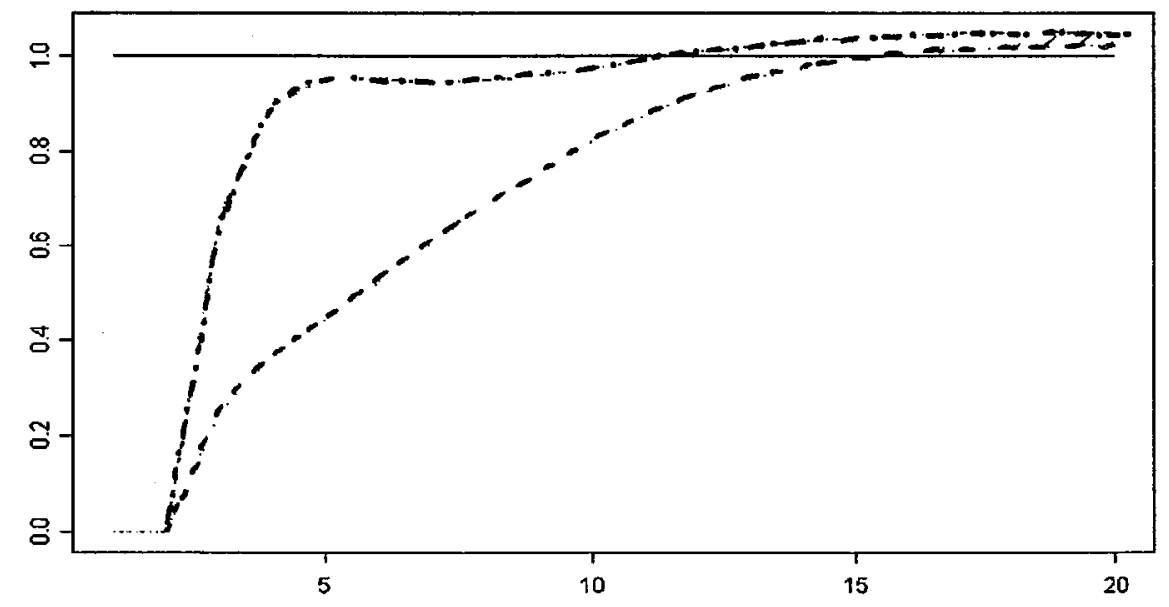

Figura 13: Gráfico da deviance da distribuição binomial para o modelo de média com e sem o fator de correção $a=0,73$ para $m=0(1) 20$ e $p=0,4$.

(- - . - . - - deviance sem correção, - - - - - = deviance com correção) 
Nota-se uma grande melhora na distribuição da deviance corrigida pelo fator $a=0,73$ para variáveis com $m$ pequeno e $p$ qualquer, e também para variáveis com $m$ grande e $p$ pequeno.

As Figuras 14 a 22 mostram os QQ-plots dos valores calculados da deviance para dados simulados de uma distribuição binomial com $m=10$ e $20 \mathrm{e}$ $p=0,1,0,2,0,3$ e 0,4 contra os quantis de uma distribuição da $\chi^{2}$ com um grau de liberdade para as variáveis sem correção e corrigidas, respectivamente.
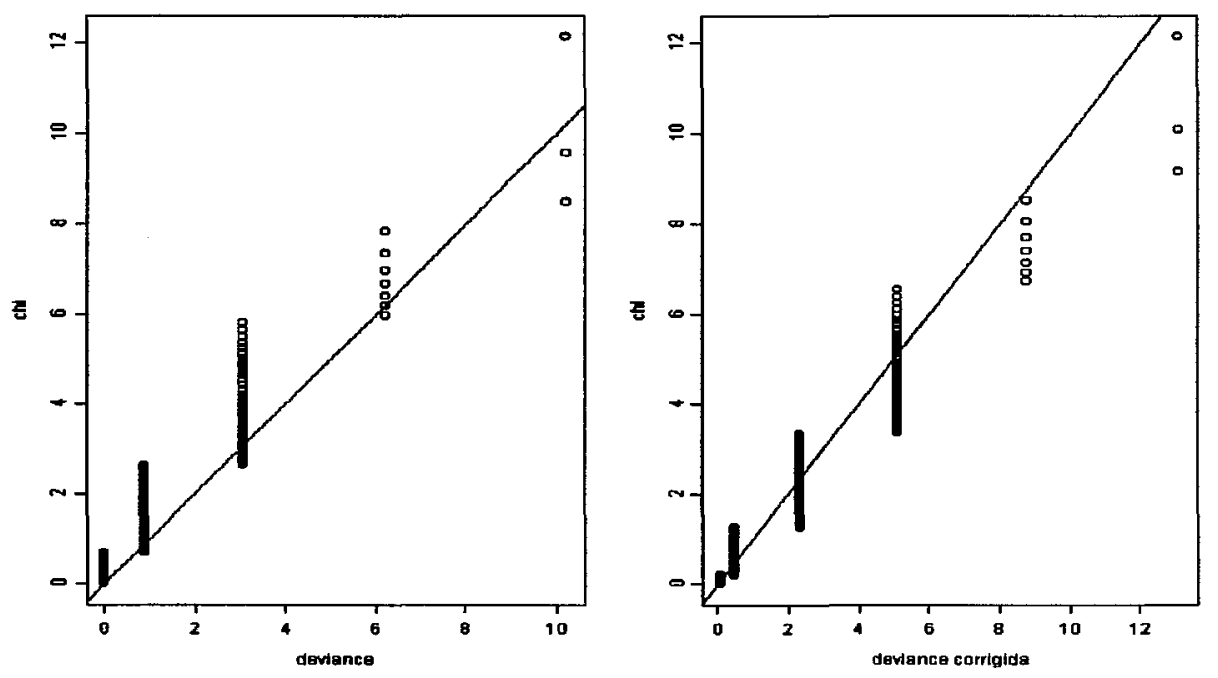

Figura 14: QQ-plots dos valores da deviance da distribuição binomial contra os valores da qui-quadrado com 1 g.l. para o modelo de média sem e com o fator de correção para $m=10$ e $p=0,1$. 

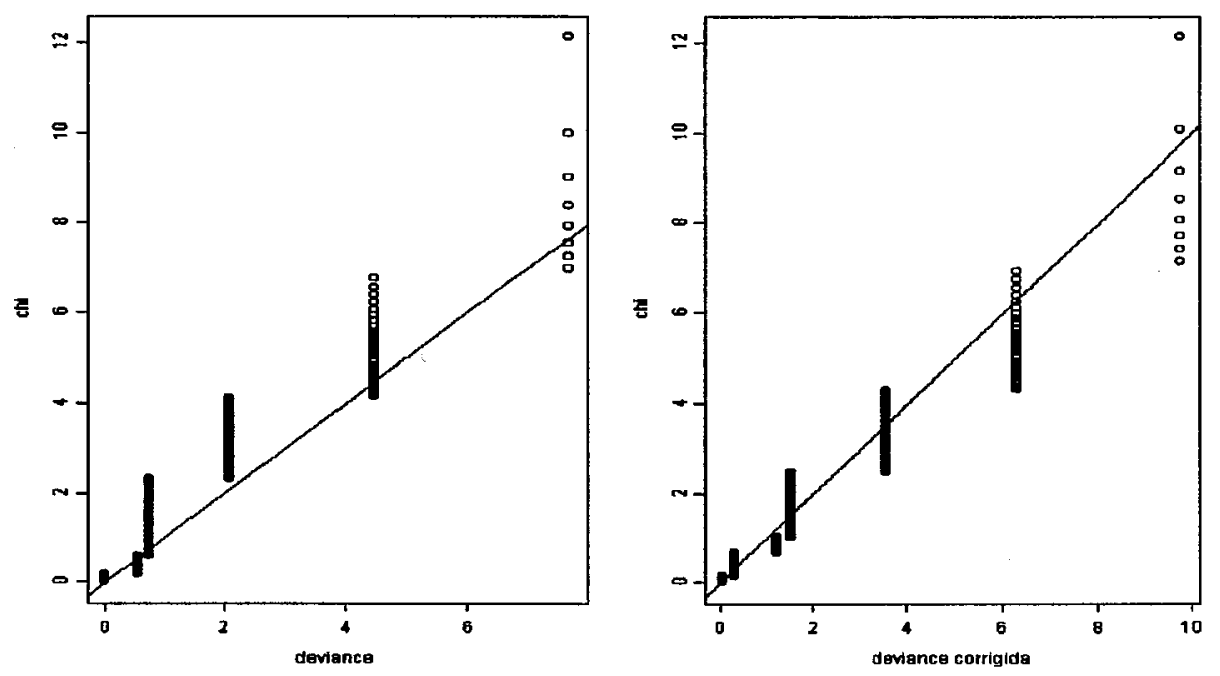

Figura 15: QQ-plots dos valores da deviance da distribuição binomial contra os valores da qui-quadrado com 1 g.l. para o modelo de média sem e com o fator de correção para $m=10$ e $p=0,2$.
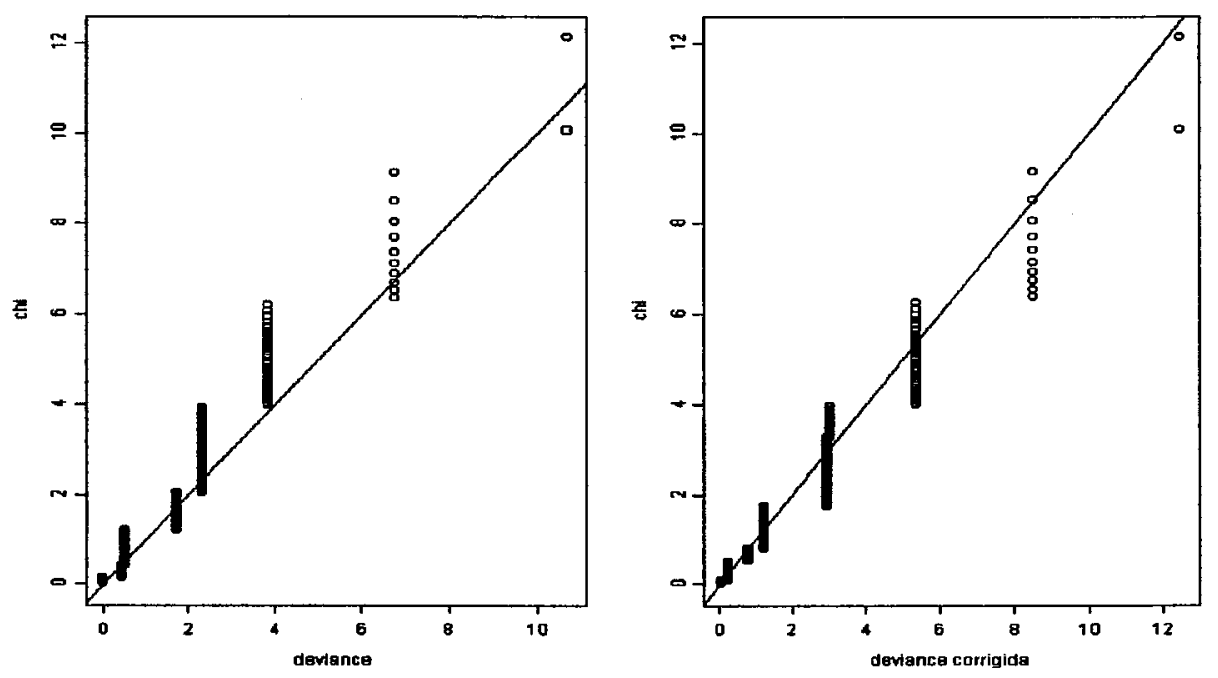

Figura 16: QQ-plots dos valores da deviance da distribuição binomial contra os valores da qui-quadrado com 1 g.l. para o modelo de média sem e com o fator de correção para $m=10$ e $p=0,3$. 

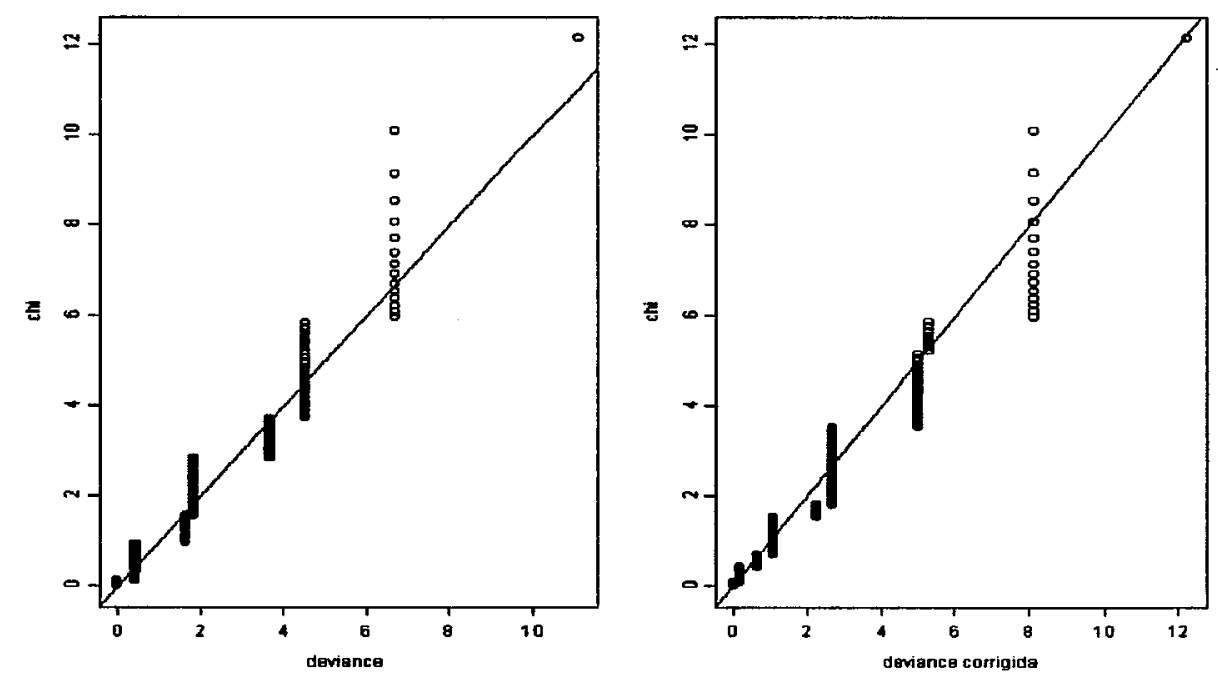

Figura 17: QQ-plots dos valores da deviance da distribuição binomial contra os valores da qui-quadrado com 1 g.l. para o modelo de média sem e com o fator de correção para $m=10$ e $p=0,4$.
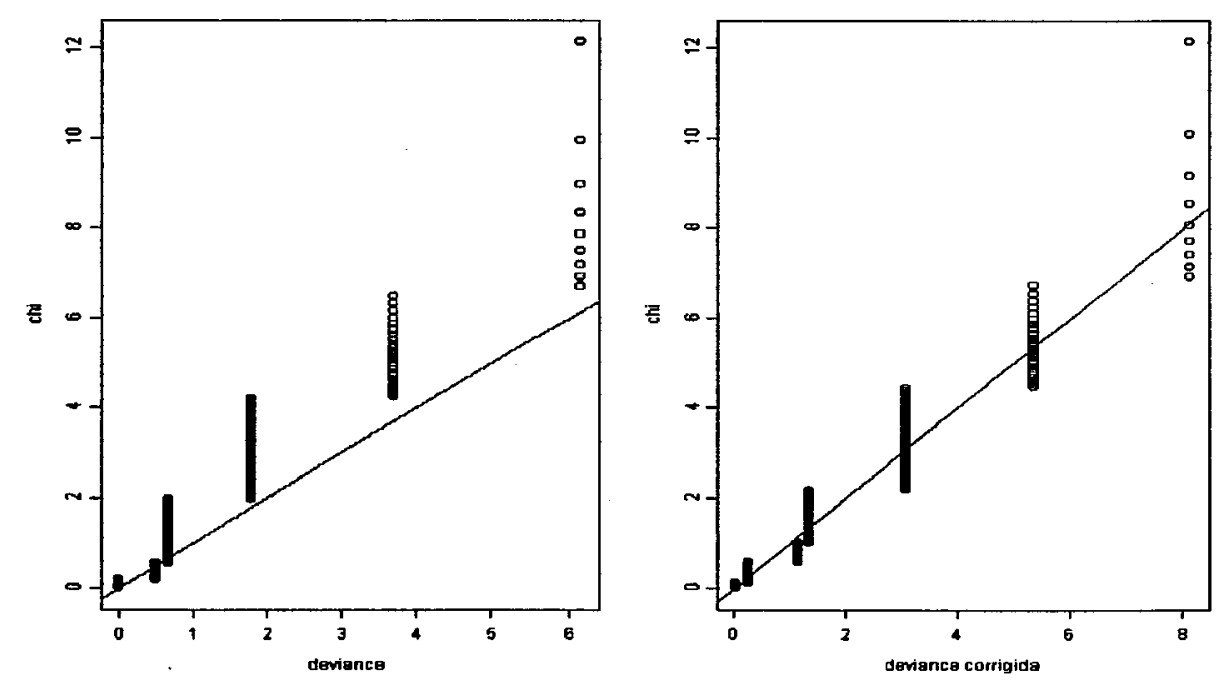

Figura 18: QQ-plots dos valores da deviance da distribuição binomial contra os valores da qui-quadrado com 1 g.l. para o modelo de média sem e com o fator de correção para $m=20$ e $p=0,1$. 

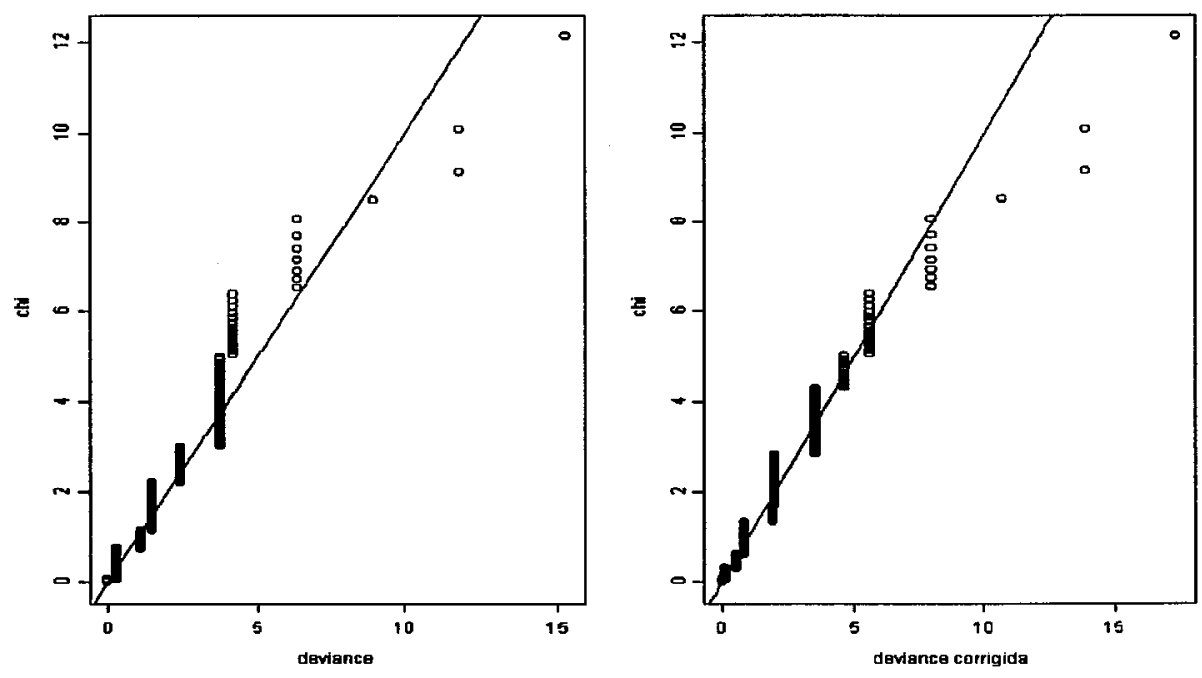

Figura 19: QQ-plots dos valores da deviance da distribuição binomial contra os valores da qui-quadrado com 1 g.l. para o modelo de média sem e com o fator de correção para $m=20$ e $p=0,2$.
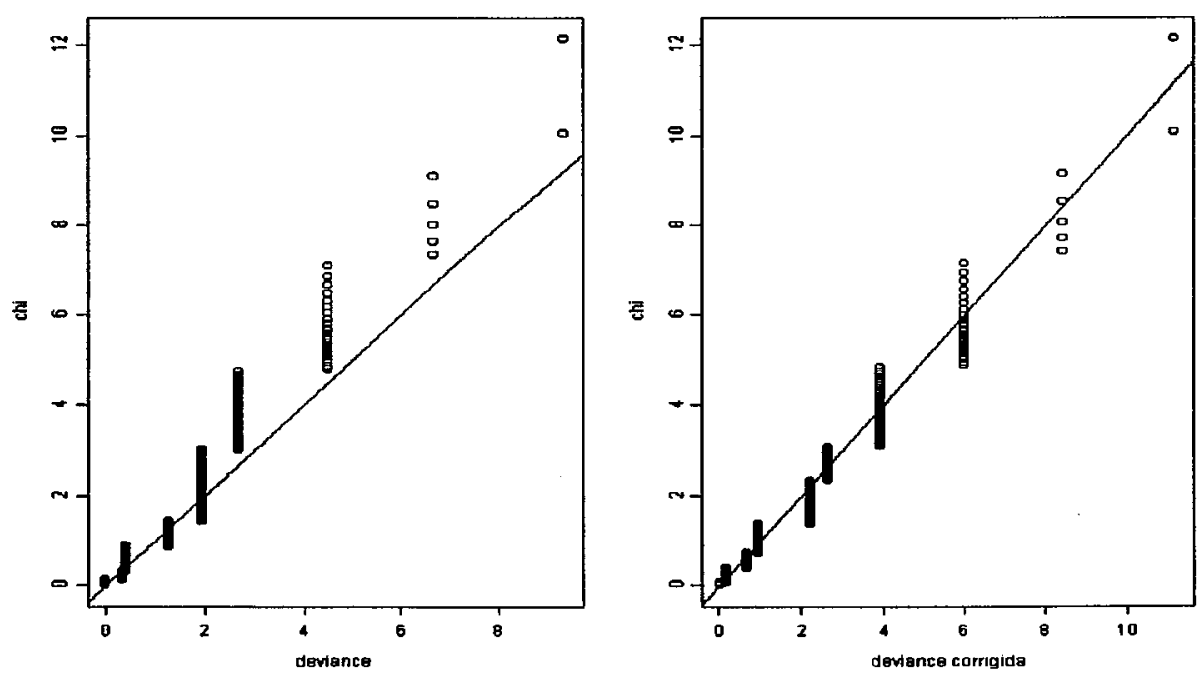

Figura 20: QQ-plots dos valores da deviance da distribuição binomial contra os valores da qui-quadrado com 1 g.l. para o modelo de média sem e com o fator de correção para $m=30$ e $p=0,1$. 

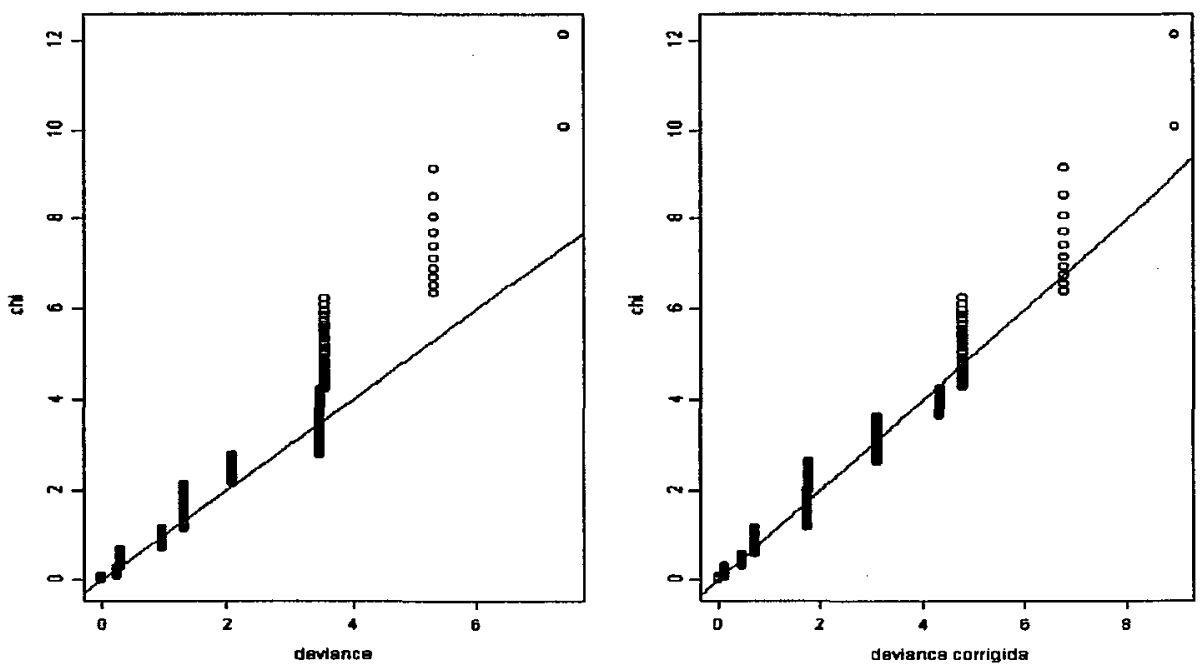

Figura 21: QQ-plots dos valores da deviance da distribuição binomial contra os valores da qui-quadrado com 1 g.l. para o modelo de média sem e com o fator de correção para $m=40$ e $p=0,1$.
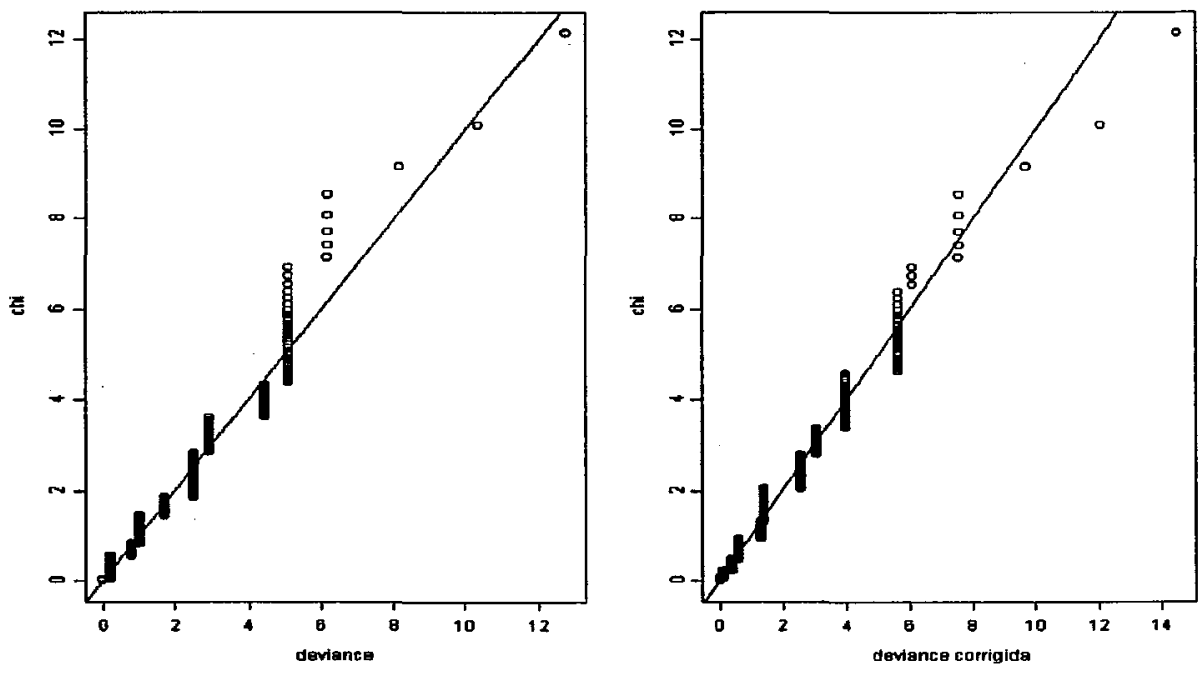

Figura 22: QQ-plots dos valores da deviance da distribuição binomial contra os valores da qui-quadrado com 1 g.l. para o modelo de média sem e com o fator de correção para $m=50$ e $p=0,1$. 
Logo, pode-se dizer que este valor é uma boa correção para as variáveis resposta que seguem uma distribuição binomial. Ainda, tem-se uma melhor aproximação inclusive para valores pequenos de $m$, já que, para valores grandes, não é necessário corrigir os dados.

Analisando as Figuras 14 a 17 para $m=10$ e $p=0,1,0,2,0,3$ e 0,4 , vê-se uma melhora no ajuste da distribuição da deviance a uma $\chi^{2}$. Como é de se esperar, quando $p=0,5$, não é preciso usar o fator de correção. O mesmo pode ser observado nas Figuras 18 e 19 quando $m=20$ e $p=0,1$ e 0,2.

Quando $m$ aumenta, não existe a necessidade de usar o fator de correção como mostram as Figuras 20 e 21.

\subsection{Estudo do Fator de Correção}

A função do fator de correção $a$ definido anteriormente, é dada por:

$$
a=\frac{1-p+p^{2}}{12 p^{2}}-\frac{1-3 p+3 p^{2}}{4 n^{2} p^{2}} .
$$

O limite de $a$ quando $n$ tende a infinito é:

$$
\lim _{n \rightarrow \infty} a=\frac{1-p+p^{2}}{12 p^{2}}
$$

que é a mesma expressão do fator para uma variável. Portanto, pode-se considerar desprezível o segundo termo do fator de correção para $n$ grande. 


\section{CONCLUSÃO}

Diante do exposto e dos resultados obtidos neste trabalho, conclui-se que

- Quando $Y$ apresenta uma distribuição binomial de parâmetros $m$ e $p$, a distribuição da deviance não pode ser aproximada pela distribuição de quiquadrado, para pequenos valores de $m$.

- Para uma variável aleatória $Y$ com distribuição binomial de parâmetros $m$ e $p$, foi obtido um fator de correção $a=0,73$, independente de $p$ que, adicionado à $Y$, aproxima a distribuição da deviance à uma distribuição $\chi^{2}$, inclusive para pequenos valores de $m$.

- Considerando $n$ variáveis aleatórias independentes e identicamente distribuídas de mesma distribuição binomial com parâmetros $m$ e $p$ e ajustando um modelo de média, o fator de correção obtido pode ser considerado o mesmo, ou seja, $a=0,73$, mostrando novamente que, adicionando esse valor à variável resposta, a distribuição da deviance se aproxima de uma distribuição $\chi^{2}$. Além disso, na presença de variáveis explanatórias, pode-se supor diferentes modelos a serem ajustados e do mesmo modo, verificar a aproximação da distribuição da deviance a uma distribuição $\chi^{2}$.

- Estudos posteriores podem ser feitos a fim de encontrar um fator de correção que possa ser utilizado para variáveis apenas independentes, ou seja, supondo números diferentes de ensaios e diferentes probabilidades de sucesso. 


\section{REFERÊNCIAS BIBLIOGRÁFICAS}

BICKEL, P.J.; DOKSUM, K.A. Mathematical Statistics. California: Holden-Day, Inc, 1977, 493p.

BLOM, G. Transformations of the binomial, negative binomial, Poisson and $\chi^{2}$ distributions. Biometrika, v.41, n.3, p.302-316, 1954.

CORDEIRO, G.M. Modelos lineares Generalizados. Campinas: VII SINAPE, 1986, 286p.

COX, D.R.; SNELL, E.J. A general definition of residuals. Journal of the Royal Statistical Society Series B, v.30, n.2, p.248-275, 1968.

COX, D.R.; SNELL, E.J. Applied Statistics. London: Chapman and Hall, 1981, 189p.

COURANT, R. Cálculo Diferencial e Integral. Rio de Janeiro: Globo, 1958.

GART, J.J.; ZWEIFEL, J.R. On the bias of various estimators of the logit and its variance with application to quantal bioassay. Biometrika, v.54, n.3, p.181-187, 1967.

JOHNSON, N.L.; KOTZ, S. Discrete Distributions. Boston: Houghton Mifflin Company, 1969, 328p. 
KAPLAN, W. Advanced Calculus. Reading, Mass.: Addison Wesley Publishing Company, 1973, 709p.

LANG, S. Cálculo. Rio de Janeiro: Livros Técnicos e Científicos Editora S.A., 1976, 366p.

LEITE, J.G.; SINGER, J.M. Métodos Assintóticos em Estatística- Fundamentos e Aplicações. In: Simpósio Brasileiro de Probabilidade e Estatística, 9.IME - USP, São Paulo, 1990, 130p.

MCCULLAGH, P.; NELDER, J.A. Generalized linear models. London: Chapman and Hall, 1991, 511p.

MEYER, P.L. Probabilidade - Aplicações à Estatística. Rio de Janeiro: Livros Técnicos e Científicos Editora S. A., 1984, 426p.

MURTEIRA, B.J.F. Probabilidades e Estatística. Portugal: McGraw-Hill, 1990, 423p.

NELDER, J.A.; WEDDERBURN, R.W.M. Generalized Linear Models. Journal of the Royal Statistical Society Series A, v.135, n.3, p.370-384, 1972.

ROUSSAS, G.G. A first course in Mathematical Statistics. Reading, Mass.: Addison-Wesley Publishing Company, 1973, 506p.

S-Plus for Windows - user's manual, 2v., StatiSci, Seattle, 1993.

TAYLOR, J.M.G.; SIQUEIRA, A.L.; WEISS, R.E. The cost of adding parameters to a model. Journal of the Royal Statistical Society Series B, v.58, n.3, p.593-607, 1996.

WEDDERBURN, R.W.M. Quasilikelihood functions, generalized linear models and the Gauss-Newton method. Biometrika, v.61, n.3, p.439-447, 1974. 